\title{
On Construction of Martin Boundaries for Second Order Elliptic Equations
}

By

\author{
Minoru Murata*
}

\section{Table of Contents}

Introduction

$\S 1$. Preliminaries

1.1. Comparison Theorem

1.2. Existence of Positive Solutions

1.3. Criticality, Subcriticality, and Minimal Growth

1.4. Martin Boundary

$\S 2$. Imbedding of a Boundary Part into the Minimal Martin Boundary

$\S 3$. Tensor Product Decomposition

$\S 4$. Proof of Theorem 3.5

$\S 5$. Schrödinger Equations in a Cone

$\S 6$. Equations in the Product of a Cone and a Bounded Domain

\$7. Direct Sum Decomposition

Appendix. A Uniqueness Theorem

References

\section{Introduction}

A generalized version of the classical Martin theorem says that any positive solution of a second order linear elliptic equation in a domain of a Riemannian manifold is represented uniquely by an integral of the Martin kernel over the Martin boundary with respect to a finite positive Borel measure which is zero at the non-minimal Martin boundary (for a precise statement, see $[22,16,34$, $29,10]$ and references therein, or Theorem 1.10 below). Therefore constructing explicitly the Martin boundary and Martin kernel is crucial in the study of positive solutions of an elliptic equation.

This paper is concerned with positive solutions of a second order elliptic equation

(0.1) $\sum_{i, j=1}^{n}-\frac{1}{\sqrt{g}} \frac{\partial}{\partial x_{i}}\left(\sqrt{g} a^{i j} \frac{\partial u}{\partial x_{j}}\right)+\sum_{i=1}^{n}\left(b^{i} \frac{\partial u}{\partial x_{i}}-\frac{1}{\sqrt{g}} \frac{\partial}{\partial x_{i}}\left(\sqrt{g} d^{i} u\right)\right)+c u=0$

Communicated by S. Matuura, February 5, 1988

* Department of Mathematics, Tokyo Metropolitan University, Fukazawa, Setagaya, Tokyo, 158 Japan. 
in a domain of a non-compact Riemannian manifold, where $g=\operatorname{det}\left(g_{i j}\right)$ with $\left(g_{i j}\right)$ being the Riemannian metric, $\left(a^{i j}\right)$ is positive definite and locally bounded, $\left(b^{i}\right)$ and $\left(d^{i}\right)$ are locally $p$-th integrable for some $p>n$, and $c$ is locally $\max (p / 2,1)$-th integrable.

The aim of this paper is to establish two methods (decomposition methods) to construct the Martin boundary and Martin kernel for such an equation as (0.1), and to explicitly construct Martin boundaries and Martin kernels for equations in unbounded domains of $R^{n}$ by applying the decomposition methods to the equations. The first method (tensor product decomposition method) is concerned with decomposition of an operator and a domain into a sum of tensor products of operators and a direct product of domains; and the second one (direct sum decomposition method) is concerned with decomposition of a domain into the union of a finite number of ends and a relatively compact set.

The problem of determining the Martin boundary and Martin kernel for a second order elliptic equation in a domain of a non-compact Riemannian manifold intrigued many mathematicians. In the case where the closure of a domain is compact in the manifold, many analysts gave sufficient conditions for the corresponding Martin boundary to be equal to the relative boundary of the domain (see $[11,12,9,39,38,17,7,35]$ ). As for the case where the closure of a domain is not compact, however, only recently has much attention been paid to the problem. Caffarelli-Littman [8] gave an elementary proof of the fact that the minimal Martin boundary for the Helmholtz operator $-\Delta+1$ on $R^{n}$ is the unit sphere $S^{n-1}$ of $R^{n}$ and the corresponding Martin kernel for $\omega$ in $S^{n-1}$ is equal to $\exp (x \omega)$ (for more general results, see [18]); which implies that any positive solution $u$ of $(-\Delta+1) u=0$ in $R^{n}$ is represented uniquely as

$$
u(x)=\int_{S^{n-1}} e^{x \omega} \mu(d \omega),
$$

where $\mu$ is a finite positive Borel measure on $S^{n-1}$. Agmon [2] gave an analogous result for a second order elliptic operator with periodic coefficients on $R^{n}$ and its extension to Riemannian covering spaces, by exploiting the translation invariance of the operator and invoking the Krein-Milman theorem. Nakai [26] showed that the Martin boundary over zero for a stationary Schrödinger equation with radial potential in a punctured disk of $\mathrm{R}^{2}$ is either one point or a unit circle (see also [14], [28] and [24]). By closely investigating Green's functions, Murata [23 and 25] explicitly constructed minimal Martin boundaries and Martin kernels for stationary Schrödinger operators $-\Delta+V$ on $R^{n}$ with potentials $V$ which are principally radial or non-radial in an extreme way. Landis-Nadirashvili [19] showed that a positive solution to a uniformly elliptic equation in a cone of $R^{n}$ which vanishes at the boundary is unique up to a constant multiple (for such uniqueness theorems, see also $[13,23,24,30,36]$ ). Aikawa [3] gave a representation formula like $(0.2)$ for positive harmonic 
functions in strips and semi-strips of $R^{n}$. Its extension to an elliptic operator with periodic coefficients and a representation formula for the operator in a convex cone in $R^{n}$ have been given recently by Pinchover [31]. On the other hand, Anderson-Shoen [6] established that the geometric boundary of a complete manifold of negative curvature is homeomorphic to its Martin boundary (see also [5]). Li-Tam [20] showed that any positive harmonic function on a complete manifold with nonnegative sectional curvature outside a compact set is a positive linear combination of a finite number of specified harmonic functions.

The rest of this paper is divided into four parts. First we introduce fundamental notions concerning positive solutions of the equation (0.1) and give relevant basic results. In Subsection 1.1 we show that a so called boundary Harnack principle holds for positive solutions of (0.1) vanishing at the boundary; the principle plays a crucial role in our study. In Subsections 1.2 and 1.3 we introduce such notions as $\delta$-positivity, minimal growth, criticality, subcriticality and minimal Green's function; and generalize some results given in [1] and [23, Section 2]. In Section 2 we show that the relative boundary of a Lipshitz domain is imbedded into the minimal Martin boundary for (0.1); which generalizes corresponding results in [16], [7] and [35].

In the second part we study positive solutions of an elliptic equation

$$
P u(x, y) \equiv\{L(x, \partial / \partial x)+W(x) A(y, \partial / \partial y)\} u(x, y)=0 \quad \text { in } \Omega_{x} \times D_{y},
$$

where $\Omega$ is a Lipshitz domain of a non-compact Riemannian manifold, $D$ is a relatively compact Lipshitz domain of another Riemannian manifold, $W(x)$ is a positive function in $\Omega, L$ and $A$ are second order elliptic differential operators as in (0.1) on $\Omega$ and $D$, respectively, and $A$ is in addition formally self-adjoint (for precise conditions, see Section 3). Denote by $\lambda_{0}<\lambda_{1} \leqq \lambda_{2} \leqq \cdots$ the eigenvalues of the Dirichlet realization $A_{D}^{\sim}$ of $A$ in $L_{2}(D)$. In Section 3 we show that there exists a minimal Green's function $G$ for $(P, \Omega \times D)$ (i. e., $(P, \Omega \times D)$ is subcritical) if and only if $\left(L+\lambda_{0} W, \Omega\right)$ is subcritical, give an "eigenfunction expansion" of $G$, and establish a method to construct the Martin boundary and Martin kernel for $(P, \Omega \times D)$. A part of a main theorem, Theorem 3.5, reads as follows.

Theorem. Assume that $\left(L+\lambda_{0} W, \Omega\right)$ is subcritical, and let $H_{j}$ be the minimal Green's functions for $\left(L+\lambda_{j} W, \Omega\right), j=0,1, \cdots$, respectively. Suppose that

(a) for each $j=1,2, \cdots$ and $\xi$ in the Martin boundary $\Sigma_{0}$ for $\left(L+\lambda_{0} W, \Omega\right)$, there exists the limit

$$
K_{\jmath}(x, \xi) \equiv \lim _{x \rightarrow \xi} H_{j}\left(x, x^{\prime}\right) / H_{0}\left(x_{0}, x^{\prime}\right),
$$

where $x_{0}$ is a fixed point in $\Omega$;

(b) the functions $K_{\jmath}(x, \xi)$ are continuous on $\Omega \times \Sigma_{0}$;

(c) $\Sigma_{0}$ is decomposed into two disjoint parts $\Sigma_{0 \infty}$ and $\Sigma_{00}$ such that 


$$
\begin{array}{lllll}
K_{3}(x, \xi)>0 & \text { on } & \Omega \times \Sigma_{0 \infty} & \text { for all } & j=1,2, \cdots \\
K_{3}(x, \xi)=0 & \text { on } & \Omega \times \Sigma_{00} & \text { for all } & j=1,2, \cdots .
\end{array}
$$

Then the Martin boundary for $(P, \Omega \times D)$ is homeomorphic to

$$
\Omega \times \partial D+\Sigma_{000} \times \bar{D}+\Sigma_{00} \times\{d\},
$$

where $d$ is an ideal point outside the closure $\bar{D}$ of $D$.

Section 4 is devoted to the proof of Theorem 3.5. A main idea of the proof is to study the asymptotic behavior of the quotient

$$
G\left(x, x^{\prime}, y, y^{\prime}\right) / H_{0}\left(x_{0}, x^{\prime}\right) \varphi_{0}\left(y^{\prime}\right),
$$

instead of $G\left(x, x^{\prime}, y, y^{\prime}\right) / G\left(x_{0}, x^{\prime}, y_{0}, y^{\prime}\right)$, as $\left(x^{\prime}, y^{\prime}\right)$ goes to infinity, where $\varphi_{0}$ is an eigenfunction associated with the smallest eigenvalue $\lambda_{0}$ of $A_{D}^{\tilde{D}}$. In Sections 5 and 6 we apply the results in Section 3 to equations in unbounded domains of $R^{n}$. Consider, for example, an elliptic operator $P=-\Delta+V(x)+U(y)$ in a strip $R_{x}^{n} \times F_{y}$, where $V$ is a real-valued radial function in $L_{q, 10 c}\left(R^{n}\right)$ for some $q>\max (n / 2,1), U$ is a real-valued function in $L_{r, 1 \text { oc }}\left(R^{m}\right)$ for some $r>\max (m / 2,1)$, and $F$ is a bounded Lipshitz domain of $R^{m}$. Let $\mu_{0}$ be the smallest eigenvalue of the Dirichlet realization of $-\Delta_{y}+U(y)$ on $L_{2}(F)$. Suppose that $\left(-\Delta_{x}+V+\right.$ $\left.\mu_{0}, R^{n}\right)$ is subcritical, and let $\Sigma$ be the Martin boundary for $\left(P, R^{n} \times F\right)$. Then Theorems $6.4 \sim 6.7$ in Section 6 imply that $\Sigma \backslash\left(R^{n} \times \partial F\right)$ is homeomorphic to

$$
\text { one point or } S^{n-1} \text { or } S^{n-1} \times \bar{F} \text {. }
$$

The results to be given in Sections 5 and 6 essentially include those given in $[8,26,14,36,3]$ and $[23$, Sections 3 and 6$]$.

In the third part we establish the direct sum decomposition method. In particular, we show in Section 7 that the Martin boundary over infinity is decomposed according decomposition of a domain near infinity: Let $\Omega$ and $\Omega_{j}$ $(j=0,1, \cdots, \nu)$ be Lipshitz domains such that $\Omega=\cup_{\nu=0}^{\nu} \Omega_{j}, \bar{\Omega}_{0}$ is compact, $\bar{\Omega}_{\imath} \cap \bar{\Omega}_{j}$ $=\phi(i \neq j ; i, j=1, \cdots, \nu)$; suppose that $(L, \Omega)$ is subcritical; then the Martin boundary $\Sigma(L, \Omega)$ for $(L, \Omega)$ is homeomorphic to

$$
\partial \Omega+\left\{\Sigma\left(L, \Omega_{1}\right) \backslash \partial \Omega_{1}\right\}+\cdots+\left\{\Sigma\left(L, \Omega_{\nu}\right) \backslash \partial \Omega_{\nu}\right\} .
$$

This implies that the Martin boundary over infinity is stable under compact perturbation in a sense. The results to be given in Section 7 are inspired by [20] and [23, Section 2].

Finally we give in Appendix a sufficient condition for an equation (0.1) in $R^{n}$ or a cone of $R^{n}$ to have a unique (up to constant multiple) positive solution vanishing at the boundary. 


\section{$\S 1$. Preliminaries}

In this section we prepare basic notions and theorems concerning positive solutions of a second order elliptic equation. The section is divided into four subsections. In Section 1.1 we shall give a comparison theorem (boundary Harnack principle) for positive solutions vanishing at boundary. In Section 1.2, on the basis of ideas in [1], we give sufficient conditions for a positive solution to exist. In Section 1.3 we shall give criteria for the criticality and subcriticality, extending results of [23, Section 2] (for related results, see also [4] and [32]). Finally, in Section 1.4 we recall the Martin theory concerning representation of positive solutions.

Let $M$ be a non-compact connected orientable Riemannian manifold of dimension $n$ and class $C^{2}$. Let $\left(g_{\imath \jmath}\right)$ be the Riemannian metric of $M, g=\operatorname{det}\left(g_{\imath \jmath}\right)$, and $d v$ the volume element of $M$. We consider a second order elliptic differential operator $L$ on $M$

$$
L=-\sum_{i, j=1}^{n} g^{-1 / 2} \partial_{\jmath}\left(g^{1 / 2} a^{i j} \partial_{\imath}\right)+\sum_{i=1}^{n}\left(b^{i} \partial_{\imath}-g^{-1 / 2} \partial_{\imath} g^{1 / 2} d_{\imath}\right)+c,
$$

where $\left(a^{i j}(x)\right),\left(b^{i}(x)\right)$ and $\left(d^{i}(x)\right)$ are real-valued measurable contravariant tensors on $M$, and $c(x)$ is a real-valued function on $M$. Here $\partial_{i}=\partial / \partial x_{2}$. We assume :

(i) $\left(a^{i j}(x)\right)$ is symmetric and positive definite for each $x$ in $M$.

(ii) For any compact set $K$ of $M$ there exist positive constants $\lambda$ and $\Lambda$ such that for any $x \in K$

$$
\lambda|\xi|^{2} \leqq \sum_{i, j=1}^{n} a_{i j}(x) \xi_{\imath} \xi_{\jmath} \leqq \Lambda|\xi|^{2} \quad \text { for all } \quad \xi \in T_{x},
$$

where $\left(a_{i j}(x)\right)=\left(a^{i_{j}}(x)\right)^{-1}, T_{x}$ is a tangent vector space of $M$ at $x$, and $|\xi|$ is the length of $\xi$ with respect to the Riemannian metric.

(iii) For some $p$ with $n<p \leqq \infty$,

$$
|b(x)|,|d(x)| \in L_{p, 10 \mathrm{c}}(M), \quad c(x) \subseteq L_{q, 1 \mathrm{oc}}(M),
$$

where $q=\max (p / 2,1), b(x)=\left(b_{1}(x), \cdots, b_{n}(x)\right)$ and $d(x)=\left(d_{1}(x), \cdots, d_{n}(x)\right)$. (Here $f \in L_{r, 10 c}(M)$ means that $f \in L_{r}(K ; d v)$ for every compact subset $K$ of $M$.)

We are interested in positive solutions of the equation

$$
L u=0 \quad \text { in } \Omega \text {, }
$$

where $\Omega$ is a domain of $M$. By a solution we mean a function $u$ in $H_{\mathrm{loc}}^{1}(\Omega)$ satisfying the equation (1.2) in the weak sense. Here and in what follows $H_{\text {loc }}^{1}(\Omega)$ and $H^{1}(\Omega)$ denote the spaces of all functions whose up to first order distributional derivatives belong to $L_{2,10 c}(\Omega)$ and $L_{2}(\Omega)$, respectively. Recall that any solution of (1.2) is Hölder continuous in $\Omega$ (see [33]). In what follows, 
$\bar{\Omega}$ and $\partial \Omega$ stand for the closure and boundary of $\Omega$ in $M$.

\subsection{Comparison Theorem}

In order to state a comparison theorem (boundary Harnack principle) and Harnack's inequality we define quantitatively a class of operators. Let $\left\{M_{k}\right\}_{k=1}^{\infty}$ be a sequence of relatively compact domains of $M$ with regular boundaries such that $\bar{M}_{k} \subset M_{k+1}$ for any $k$ and $\bigcup_{k=1}^{\infty} M_{k}=M$. Let $\left\{\lambda_{k}, \Lambda_{k}, N_{k}\right\}_{k=1}^{\infty}$ be a sequence in $R_{+}^{3}$.

Definition 1.1. We say that $L$ belongs to $\mathcal{E}\left\{\lambda_{k}, \Lambda_{k}, N_{k}\right\}$ if $L$ is an operator of the form (1.1) satisfying (i), (ii), (iii), and the following inequalities

$$
\begin{aligned}
& \lambda_{k}|\xi|^{2} \leqq \sum_{i, j=1}^{n} a_{i_{j}}(x) \xi_{2} \xi_{j} \leqq \Lambda_{k}|\xi|^{2} \quad \text { for all } \quad x \in M_{k} \text { and } \xi \Subset T_{x}, \\
& \||b|+|d|\|_{L_{p}\left(M_{k}\right)}+\|c\|_{L_{q}\left(M_{k}\right)} \leqq N_{k} .
\end{aligned}
$$

The following theorem follows from a result of [33].

Theorem 1.2 (Harnack's inequality). Let $L$ belong to $\mathcal{E}\left\{\lambda_{k}, \Lambda_{k}, N_{k}\right\}$, and $K$ a compact subset of an open set $\Omega$ of $M$. Then there exists a positive constant $C$ depending only on $\mathcal{E}\left\{\lambda_{k}, \Lambda_{k}, N_{k}\right\}, K$, and $\Omega$ such that

$$
\max _{K} u \leqq C \min _{K} u
$$

for any nonnegative solution $u$ of (1.2).

We call a domain $D$ of $M$ is a Lipshitz domain if for each point $z$ of $\partial D$ there exist a coordinate neighborhood $(U, \phi)$ of $z$, a ball $B$ in $U$ with center $z$, a function $\varphi$ on $R^{n-1}$, and a positive constant $m$ such that

$$
\begin{aligned}
& \psi(B \cap D)=\psi(B) \cap\left\{\left(x^{\prime}, x_{n}\right) ; x^{\prime} \in R^{n-1}, x_{n}>\varphi\left(x^{\prime}\right)\right\}, \\
& \phi(B \cap \partial D)=\psi(B) \cap\left\{\left(x^{\prime}, \varphi\left(x^{\prime}\right)\right) ; x^{\prime} \in R^{n-1}\right\}, \\
& \left|\varphi\left(x^{\prime}\right)-\varphi\left(y^{\prime}\right)\right| \leqq m\left|x^{\prime}-y^{\prime}\right| \quad \text { for any } \quad x^{\prime}, y^{\prime} \in R^{n-1}
\end{aligned}
$$

(cf. [7]). By definition, a domain without boundary is also a Lipshitz domain. We denote by $B(r, z)$ a ball of radius $r$ centered at $z$.

The following theorem is an extension of Theorem 1.4 of [7], where uniformly elliptic operators of divergence form on $R^{n}$ were treated (see also [9] and [39]).

Theorem 1.3 (Comparison theorem). Let $D$ be a Lipshitz domain of $M$, $z \Subset \partial D$, and $L$ an operator in $\mathcal{E}\left\{\lambda_{k}, \Lambda_{k}, N_{k}\right\}$. Then there exist positive constants 
$r_{0}$ and $C$ depending only on $\mathcal{E}\left\{\lambda_{k}, \Lambda_{k}, N_{k}\right\}$ and the Lipshitz continuity of $\partial D$ near $z$ such that for any $r$ with $0<r<r_{0}$ and any positive solutions $u$ and $v$ of $L u=0$ in $B(8 r, z) \cap D$ which vanish continuously on $B(8 r, z) \cap \partial D$

$$
u(x) / u\left(A_{r}(z)\right) \leqq C v(x) / v\left(A_{r}(z)\right) \quad \text { for all } x \in B(r, z) \cap D .
$$

Here $A_{r}(z)$ is a point in $\partial B(r, z) \cap D$ whose distance from $z$ is uniformly proportional to $r$, i.e., there exists a positive constant $\delta$ such that $\delta r \leqq \operatorname{dist}\left(A_{r}(z), z\right) \leqq r / \delta$ for any $0<r<r_{0}$.

Proof. Since the theorem is of local character, we may assume that $\bar{D}$ is compact. Then we can choose an open neighborhood $W$ of $\partial D$ so small that all $L$ in $\varepsilon\left\{\lambda_{k}, \Lambda_{k}, N_{k}\right\}$ are cocercive on the Sobolev space $H_{0}^{1}(W)$. Solving the Dirichlet problem and using Harnack's inequality (cf. [33] and [1]), we can get a positive solution $h$ of $L h=0$ in $W$ such that

$$
1 / c<h(x)<c \quad \text { for all } x \in \bar{V},
$$

where $V$ is an open set with $\partial D \subset V \subset \bar{V} \subset W$ and $c$ is a positive constant depending only on $W, V$ and $\mathcal{E}\left\{\lambda_{k}, \Lambda_{k}, N_{k}\right\}$. Let $r_{1}=\operatorname{dist}(\partial D, \partial V)$, and $u$ a positive solution as in the theorem with $8 r<r_{1}$. Put $u_{h}=u / h$ and $L_{h}=h L h$. Then

$$
L_{h}=-\sum_{i, j=1}^{n} g^{-1 / 2} \partial_{\jmath}\left(h^{2} g^{1 / 2} a^{\imath \jmath} \partial_{\imath}\right)+\sum_{\imath=1}^{n} h^{2}\left(b^{i}-d^{\imath}\right) \partial_{\imath},
$$

and $u_{h}$ is a positive solution of $L_{h} u_{h}=0$ in $B(8 r, z) \cap D$ which vanishes continuously on $B(8 r, z) \cap \partial D$. Note that $L_{h}$ belongs to the class $\mathcal{E}\left\{c^{2} \lambda_{k}, c^{-2} \Lambda_{k}, c^{2} N_{k}\right\}$ with the whole space $M$ replaced by $W$. Thus, by applying results of [33] and slightly modifying the argument in the proof of Theorem 1.4 of [7], we get positive constants $r_{0}<r_{1}$ and $C^{\prime}$ depending only on $\mathcal{E}\left\{c^{2} \lambda_{k}, c^{-2} \Lambda_{k}, c^{2} N_{k}\right\}$ and the modulus of Lipshitz continuity of the boundary such that for any $r$ with $0<r<r_{0}$

$$
u_{h}(x) / u_{h}\left(A_{r}(z)\right) \leqq C^{\prime} v_{h}(x) / v_{h}\left(A_{r}(z)\right) \quad \text { for all } \quad x \in B(r, z) \cap D .
$$

This yields (1.5) with $C=c^{4} C^{\prime}$.

Q.E.D.

\subsection{Existence of Positive Solutions}

Let $D$ be a relatively compact domain of $M$, and $L$ the operator (1.1). Denote by $H_{0}^{1}(D)$ the Hilbert space defined by the completion of $C_{0}^{1}(D)$ in $H^{1}(D)$. Let $L_{D}$ be a linear operator in $L_{2}(D)$ defined by:

$$
L_{D} u=L u \quad \text { for } \quad u \in \operatorname{Domain}\left(L_{D}^{\tilde{D}}\right)=\left\{u \in H_{0}^{1}(D) ; L u \in L_{2}(D)\right\} \text {. }
$$

We call $L_{\bar{D}}^{\tilde{D}}$ the Dirichlet realization of $L$ in $L_{2}(D)$. Denote by $\sigma\left(L_{\tilde{D}}\right)$ the spectrum of $L_{D}$. Put 


$$
\Gamma(L, D)=\inf \left\{\operatorname{Re} z ; z \in \sigma\left(L_{\tilde{D}}\right)\right\} .
$$

We say that $u$ is a supersolution of the equation $L v=0$ in $D$ if $u$ is a realvalued function in $H_{\mathrm{loc}}^{1}(D)$ such that $L u \geqq 0$ in $D$ in the distribution sense. A fundamental fact essentially shown by Agmon [1] is :

$\Gamma(L, D)>0$ if and only if $L$ is $\delta$-positive in $D$, i.e., if $u$ is a supersolution of $L v=0$ in $D$ and $u \geqq 0$ on $\partial D$, then $u \geqq 0$ in $D$.

The following theorem generalizes a fact well-known in the special case where $L_{D}$ is self-adjoint.

Theorem 1.4. Suppose that $D$ is a relatively compact domain of $M$. Then $\Gamma(L, D)$ is a simple eigenvalue with positive eigenfunction $\varphi$. Furtiermore, any positive supersolution of (1.2) with $\Omega=D$ is a constant multiple of $\varphi$.

Proof. For the sake of completeness we shall give a proof. We first obtain along the line given in $[1, \mathrm{p} .29]$ that $\Gamma(L, D)$ is an eigenvalue. We next construct a positive eigenfunction. For $\lambda<\Gamma \equiv \Gamma(L, D)$, solve the Dirichlet problem

$$
u_{\lambda}-1 \Subset H_{0}^{1}(D), \quad(L-\lambda) u_{\lambda}=0 \text { in } D .
$$

Then $u_{\lambda}=1-G_{\lambda}((L-\lambda) \cdot 1)$, where $G_{\lambda}$ is Green's operator. By the $\delta$-positivity, $u_{\lambda}>0$ in $D$. Put $v_{\lambda}=u_{\lambda} /\left\|u_{\lambda}\right\|$, where $\left\|u_{\lambda}\right\|$ is the norm of $u_{\lambda}$ in $L_{2}(D)$. Then we can choose a sequence $\left\{\lambda_{j}\right\}_{j=1}^{\infty}$ such that as $j \rightarrow \infty$

$$
\lambda_{j} \rightarrow \Gamma, \quad\left\|u_{\lambda_{j}}\right\| \rightarrow \infty, \quad v_{\lambda_{j}}-1 /\left\|u_{\lambda_{j}}\right\| \rightarrow \varphi \text { weakly in } H_{0}^{1}(D)
$$

for some $\varphi$ in $H_{0}^{1}(D)$. Clearly, $\varphi$ is a positive solution of the equation $(L-\Gamma) \varphi$ $=0$ in $D$. Let $u$ be any eigenfunction for $\Gamma$, and $u_{+}=\max (u, 0)$. We may assume that $u_{+} \neq 0$. Then $(L-\Gamma) u_{+} \leqq 0$ in $D$ and $u_{+} \in H_{0}^{1}(D)$. Since $L$ is coercive on $H_{0}^{1}(E)$ with $E$ being the intersection of $D$ and a sufficiently small neighborhood of $\partial D$, there exists a positive constant $\varepsilon$ such that $\varphi-\varepsilon u_{+}>0$ in $D$. Put $\varepsilon_{0}=\sup \left\{\varepsilon>0 ; \varphi-\varepsilon u_{+}>0\right.$ in $\left.D\right\}$. Then we see from the maximality that $\varphi=\varepsilon_{0} u_{+}$. Hence $u=u_{+}=\varphi / \varepsilon_{0}$, which completes the proof of the iirst half of the theorem. The second half can be shown similarly.

Q.E.D.

Now, let $\Omega$ be a domain of $M$ whose closure $\bar{\Omega}$ may not be compact. Let

$$
\Gamma(L, \Omega)=\inf \{\Gamma(L, D) ; D \subset \Omega, D \Subset M\} .
$$

Here $D \Subset M$ means that $D$ is a subdomain of $M$ whose closure is compact in $M$. We note that (1.8) and (1.9) are consistent, since $\Gamma(L, D) \geqq \Gamma\left(L, D^{\prime}\right)$ for $D \subset D^{\prime} \Subset M$ (see [1, Remark 3.5], which also implies that (1.9) is equal to the one defined by Agmon [1, (3.11)]). The following theorem is a slight extension of Theorem 3.1 of [1]. 
Theorem 1.5. The following properties are equivalent.

(i) There exists a positive solution of (1.2).

(ii) There exists a positive supersolution of (1.2).

(iii) $\Gamma(L, \Omega) \geqq 0$.

(iv) $\Gamma(L, D)>0$ for every open set $D \Subset M$ with $D \subset \Omega$ and $\partial D \cap \Omega \neq \phi$.

(v) $L$ is $\delta$-positive in such an open set $D$ as in (iv).

Proof. By employing the argument in the proof of Theorem 3.1 in [1] with minor modification, we get the equivalence of (i), (ii) and (iii) and that of (iv) and (v). Since (iv) clearly implies (iii), it suffices to show that (i) implies (iv). Let $u$ be a positive solution of $L u=0$ in $\Omega$. Since $u$ is also a positive solution of $L u=0$ in $D, \Gamma(L, D) \geqq 0$. Suppose that $\Gamma(L, D)=0$. Then we obtain by Theorem 1.4 that there exist a positive constant $C$ and a positive solution $\varphi$ in $H_{0}^{1}(D)$ of $L \varphi=0$ in $D$ such that $u=C \varphi$. But $u>0$ on $\partial D \cap \Omega$, which is a contradiction. Hence $\Gamma(L, D)>0$.

Q.E.D.

\subsection{Criticality, Subcriticality, and Minimal Growth}

Let $\Omega$ be a domain of $M$, and $L$ the operator (1.1). Assume that $\Gamma(L, \Omega)$ $\geqq 0$. Choose an increasing sequence of domains $\left\{\Omega_{k}\right\}_{k=1}^{\infty}$ such that $\Omega_{k} \Subset M$, $\partial \Omega_{k} \cap \Omega \neq \phi, \Omega_{k} \subset \Omega$, and $\bigcup_{k=1}^{\infty} \Omega_{k}=\Omega$. Let $G_{k}$ be the Green's function associated with $L_{\Omega_{k}}$. By Theorem 1.5 and Harnack's inequality, the sequence $G_{k}(\cdot, y)$ is increasing as $k \rightarrow \infty$ and either it converges to an $L_{1,10 c}(\Omega)$-function $G(\cdot, y)$ or it diverges to infinity. In the first case, $G(\cdot, y)$ is positive in $\Omega$ and clearly satisfies $L G(\cdot, y)=\delta_{y}(\cdot)$, where $\delta_{y}(\cdot)$ is Dirac's measure concentrated at $y \in \Omega$. We call $G(\cdot, y)$ the minimal Green's function for $L$ in $\Omega$. Obviously, whether a minimal Green's function exists or not is independent of the choice of an exhaustion $\left\{\Omega_{k}\right\}$ of $\Omega$. We call $(L, \Omega)$ subcritical in the first case, and critical in the second case (cf. [23]). (We call $(L, \Omega)$ supercritical if $\Gamma(L, \Omega)<0$.)

Let $\delta \Omega$ be the boundary of a compactification $\Omega^{\wedge}$ of $\Omega$, and $\gamma$ a subset of $\delta \Omega$. Suggested by Agmon [1] we call $u$ a solution of minimal growth at $\gamma$ if $u$ has the following properties (i) and (ii):

(i) $u$ is a solution of the equation $L u=0$ in the intersection of $\Omega$ and a neighborhood of $\gamma$ in $\Omega^{\wedge}$ (which we call a $\Omega$-neighborhood of $\gamma$ ).

(ii) For any positive solution $v$ of $L v=0$ in a $\Omega$-neighborhood of $\gamma$ there exists a positive constant $C$ such that $|u| \leqq C v$ in another $\Omega$-neighborhood.

From the proof of Theorems 5.2 and 5.4 in [1] and the definition of criticality we have the following theorem.

Theorem 1.6. ( $L, \Omega)$ is critical if and only if there exists a positive solution $u$ of (1.2) of minimal growth at infinity of the one point compactification of $\Omega$. In this case, any positive supersolution of (1.2) is a constant multiple of $u$. 
The following theorem is an extension of Theorem 2.4 of [23] (see also $[15$, Theorem 2] and [4, Theorem 2]).

Theorem 1.7. (i) $(L, \Omega)$ is critical if and only if $\Gamma(L, \Omega) \geqq 0$ and $\Gamma(L-r, \Omega)<0$ for any nonnegative function $r$ in $L_{p, 10 c}(\Omega)$ which is positive on a set of positive measure.

(ii) If $\Gamma(L-r, \Omega) \geqq 0$ for some $r$ as in (i), then $(L, \Omega)$ is subcritical. Conversely, if $(L, \Omega)$ is subcritical, then there exist a strictly positive continuous function $s$ on $\Omega$ such that $\Gamma(L-s, \Omega) \geqq 0$.

Proof. We have only to show (ii), since (i) follows from (ii). First, assume that $\Gamma(L-r, \Omega) \geqq 0$. Then, by Theorem 1.5 , there exists a positive solution $v$ of $(L-r) v=0$ in $\Omega$. Clearly, $v$ is a positive supersolution of $L u=0$ in $\Omega$. Now, suppose that $(L, \Omega)$ is not subcritical. Then $(L, \Omega)$ must be critical. By Theorem 1.6, $v=C u$ for a constant $C$ and a positive solution $u$ of (1.2). This is a contradiction. Next, assume that $(L, \Omega)$ is subcritial. We see that for any nonnegative continuous function $f$ with compact support in $\Omega$ there exists a positive solution $u$ of $L u=f$ in $\Omega$. Therefore, by using a partition of unity, we can construct (cf. the proof of Theorem 2 in [4]) a positive continuous function $g$ on $\Omega$ such that $L g$ is strictly positive continuous function on $\Omega$. Put $s=L g / g$. Then $g$ is a solution of $(L-s) g=0$, which implies that $\Gamma(L-s, \Omega) \geqq 0$.

The following theorem is an extension of Theorem 2.6 in [23].

Theorem 1.8. Let $\Omega^{\prime}$ be a domain of $M$ such that $\Omega^{\prime} \subset \Omega$ and $\Omega \backslash \bar{\Omega}^{\prime} \neq \phi$. If $\Gamma(L, \Omega) \geqq 0$, then $\left(L, \Omega^{\prime}\right)$ is subcritical.

Proof. Choose a nonnegative continuous function $r \neq 0$ with support in $\Omega \backslash \bar{\Omega}^{\prime}$. By Theorem 1.7, $(L+r, \Omega)$ is subcritical ; and so there exists a minimal Green's function $H$ for $L+r$ in $\Omega$. Let $\left\{\Omega_{k}^{\prime}\right\}_{k=1}^{\infty}$ be an exhaustion of $\Omega^{\prime}$, and $G_{k}^{\prime}$ Green's function for $L_{\Omega_{k}^{\prime}}$. By Theorem 1.5, $G_{k}^{\prime} \leqq H$ for all $k=1,2, \cdots$. Thus $\left(L, \Omega^{\prime}\right)$ is subcritical.

Q.E.D.

We close this subsection with the following theorem, an extension of the formula (2.8) in [23].

Theorem 1.9. Assume that $(L, \Omega)$ is critical. Let $r \neq 0$ be a nonnegative continuous function with compact support in $\Omega$. Let $G$ be the minimal Green's function for $L+r$ in $\Omega$. Then a positive salution $u$ of (1.2) satisfies

$$
u(x)=\int G(x, y)(r u)(y) d y .
$$


Proof. With $G_{k}$ being the Green's function for $\left(L+r, \Omega_{k}\right), k=1,2, \cdots$, put $v_{k}(x)=\int G_{k}(x, y)(r u)(y) d y$. Then, $(L+r)\left(u-v_{k}\right)=0$ in $\Omega_{k}$ and $u-v_{k}>0$ on $\partial \Omega_{k}$. Thus $u \geqq v_{k}$ in $\Omega_{k}$ for any $k$, which implies that $u \geqq v$ in $\Omega$ with $v$ being the right hand side of (1.10). We have that $L v=r(u-v) \geqq 0$. By Theorem 1.6, $v=C u$ for some $C>0$; and so $0=L v=r(u-v)$. Hence $v=u$ Q.E.D.

\subsection{Martin Boundary}

Suppose that $(L, \Omega)$ is subcritical, and let $G$ be the associated minimal Green's function. Then we see that Martin's representation theorem of positive harmonic functions can be extended to positive solutions of the equation (1.2) (cf. $[22,34,16,10]$; see also [29]). For completeness, we explain briefly the representation theorem. Fix a point $x_{0}$ in $\Omega$. For $x$ and $y$ in $\Omega$, put

$$
K(x, y)=G(x, y) / G\left(x_{0}, y\right) \quad \text { if } \quad x_{0} \neq y,
$$

$K(x, y)=0$ if $x_{0}=y$ and $x \neq y$, and $K(x, y)=1$ if $x_{0}=y=x$. A sequence $\left\{y_{k}\right\}_{k=1}^{\infty}$ is called a fundamental sequence if $\left\{y_{k}\right\}$ has no point of accumulation in $\Omega$ and $\left\{K\left(\cdot, y_{k}\right)\right\}_{k=1}^{\infty}$ converges to a positive solution of the equation (1.2). Two fundamental sequences $\left\{y_{k}\right\}$ and $\left\{y_{k}^{\prime}\right\}$ are called equivalent if $\lim _{k \rightarrow \infty} K\left(\cdot, y_{k}\right)=$ $\lim _{k \rightarrow \infty} K\left(\cdot, y_{k}^{\prime}\right)$. Denote by $\Sigma$ the set of all equivalence classes of fundamental sequences. For $\omega$ in $\Sigma$, put $K(\cdot, \omega)=\lim _{k \rightarrow \infty} K\left(\cdot, y_{k}\right)$ with $\left\{y_{k}\right\}$ being a representative of $\omega$. Recall that a positive solution $u$ of (1.2) is called minimal if for any positive solution $v$ of (1.2) with $v \leqq u$ in $\Omega$ there exists a positive constant $C$ such that $v=C u$ in $\Omega$. Let $\sigma$ be the set of all $\omega$ in $\Sigma$ such that $K(\cdot, \omega)$ is a minimal solution. Denote by $\bar{\Omega}^{L}$ the disjoint union $\Omega+\Sigma$ of the sets $\Omega$ and $\Sigma$. We call $\Sigma, \sigma, \bar{\Omega}^{L}$, and $K(\cdot, \omega)$ with $\omega \in \Sigma$ the Martin boundary, minimal Martin boundary, Martin compactification of $\Omega$, and Martin kernel for $(L, \Omega)$ with reference point $x_{0}$, respectively. Choose a positive continuous function $f$ belonging to $L_{1}(\Omega)$, and set

$$
d\left(z, z^{\prime}\right)=\int_{\Omega} \min \left(1,\left|K(x, z)-K\left(x, z^{\prime}\right)\right|\right) f(x) d v_{x}
$$

for any $z$ and $z^{\prime}$ in $\bar{\Omega}^{L}$. Then it is easily seen that $d$ is a metric on $\bar{\Omega}^{L}$; which is compact with respect to the topology induced by the metric $d$; the relative topology of $\Omega$ is equal to the original one; and $\Sigma$ is the boundary of $\bar{\Omega}^{L}$. Furthermore, we have

Theorem 1.10. (i) Any minimal solution of (1.2) is equal to a constant multiple of $K(\cdot, \omega)$ for some $\omega$ in $\sigma$.

(ii) $\sigma$ is a countable intersection of open subsets of $\Sigma$.

(iii) $K(x, z)$ is continuous on $\left(\Omega \times \bar{\Omega}^{L}\right) \backslash\{(x, x) ; x \equiv \Omega\}$.

(iv) For any positive solution $u$ of (1.2) there exists a unique finite non- 
negative Borel measure $\mu$ on $\Sigma$ such that $\mu(\Sigma \backslash \sigma)=0$ and

$$
u(x)=\int_{\sigma} K(x, \omega) \mu(d \omega) .
$$

\section{$\S 2$. Imbedding of a Boundary $\mathbb{P}$ art into the Minimal Martin Boundary}

Let $\Omega$ be a domain of $M$, and $L$ be the operator (1.1) on $M$. Assume that $(L, \Omega)$ is subcritical. Let $\Sigma, \sigma, \bar{\Omega}^{L}$, and $K(\cdot, \omega)$ with $\omega \in \Sigma$ be the Martin boundary, minimal Martin boundary, Martin compactification of $\Omega$, and Martin kernel for $(L, \Omega)$. A main result of this section is the following theorem, which considerably extends Theorem 6.1 of [16] where more regularity of $\partial \Omega$ and the coefficients of $L$ was assumed.

Theorem 2.1. Assume that $(L, \Omega)$ is subcritical. Let $S$ be an open subset of $\partial \Omega$ such that $S \subset \partial D$ for some Lipshitz domain $D$ with $D \subset \Omega$. Then $S$ is homeomorphically imbedded into $\sigma$; more precisely, for any point $z$ in $S$ there corresponds a point $\omega_{z}$ in $\sigma$ and the mapping $\Phi$ defined by

$$
\Phi(x)=x \text { for } x \in \Omega \text { and } \Phi(z)=\omega_{z} \text { for } z \in S
$$

gives a homeomorphism of $\Omega+S$ as a subspace of $M$ onto $\Omega+\left\{\omega_{z} ; z \equiv S\right\}$ as a subspace of $\bar{\Omega}^{L}$.

We prepare a lemma for the proof of Theorem 2.1.

Lemma 2.2. Let $\mathcal{F}$ be a non-empty family of continuous functions on $\Omega$ having the following properties (i) (iii):

(i) Any $u$ in I satisfies

$$
L u=0 \text { and } u>0 \text { in } \Omega, u\left(x_{0}\right)=1 \text {, }
$$

where $x_{0}$ is a fixed point in $\Omega$.

(ii) If $u, v \in \mathscr{I}$ and $u>v$ in $\Omega$, then

$$
(u-v)(\cdot) /(u-v)\left(x_{0}\right) \in \mathscr{I} .
$$

(iii) There exist a positive constant $C$, an increasing sequence $\left\{O_{k}\right\}_{k=1}^{\infty}$ of open subsets of $\Omega$ with $\cup_{k=1}^{\infty} O_{k}=\Omega$, and a sequence $\left\{x_{k}\right\}_{k=1}^{\infty}$ of points with $x_{k} \in$

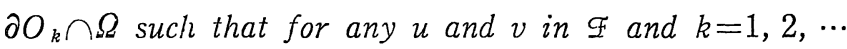

$$
\begin{aligned}
& u(x) / u\left(x_{k}\right) \leqq C v(x) / v\left(x_{k}\right) \text { for all } x \in \partial O_{k} \cap \Omega, \\
& \text { if } u \leqq C^{\prime} v \text { on } \partial O_{k} \cap \Omega \text { for some } C^{\prime}>0 \text {, then } \\
& u \leqq C^{\prime} v \text { in } O_{k} .
\end{aligned}
$$

Then $\mathcal{F}$ consists of only one element. 
Remark. Conversely, if a family $\mathscr{F}$ satisfying (i) consists of one point, then (ii) and (iii) are clearly satisfied.

Proof. Let $u$ and $v$ be elements in $\mathscr{I}$. By (2.1), we get

$$
1=u\left(x_{0}\right) \leqq\left[C u\left(x_{k}\right) / v\left(x_{k}\right)\right] v\left(x_{0}\right)=C u\left(x_{k}\right) / v\left(x_{k}\right) \text { for any } k .
$$

Thus $u \leqq C^{2} v$ in $\Omega$. Put $\varepsilon_{0}=\sup \{\varepsilon>0 ; \varepsilon u<v$ in $\Omega\}$ and $w=v-\varepsilon_{0} u$. Then, either $w=0$ or $w>0$ in $\Omega$. Suppose that $w>0$ in $\Omega$. By (i), $w(x) / w\left(x_{0}\right)$ belongs to $\mathscr{F}$. Thus the same argument as above shows that $w>\delta u$ in $\Omega$ for some $\delta>0$, which contradicts the maximality of $\varepsilon_{0}$. Hence $w=0$, and so $v=\varepsilon_{0} u$.

Q.E.D.

Proof of Theorem 2.1. Let $z$ be a point in S. By Harnack's inequality, there exists a sequence $\left\{y_{k}\right\}_{k=1}^{\infty}$ such that $y_{k} \rightarrow z$ and $K\left(\cdot, y_{k}\right) \rightarrow h(\cdot)$ as $k \rightarrow \infty$ with $h$ being a positive solution of (1.2). We see that $h$ has the following properties :

(a) $L h=0$ and $h>0$ in $\Omega, h\left(x_{0}\right)=1$.

(b) Let $M^{\wedge}$ be a one point compactification of $M$. Let $\delta \Omega$ be the boundary of the closure of $\Omega$ in $M^{\wedge}$. Then $h$ is of minimal growth at $\delta \Omega \backslash B(r, z)$ for any $r>0$, where $B(r, z)$ is a ball of radius $r$ centered at $z$. (As for the minimal growth, see Section 1.3.)

We claim that the set $\mathscr{F}$ of all functions satisfying (a) and (b) consisis of one point. Properties (i) and (ii) in Lemma 2.2 hold clearly. For $k=1,2, \cdots$, put $O_{k}=\Omega \backslash B\left(r_{0} / 2 k, z\right)$ and $x_{k}=A_{r_{0} / 2 k}(z)$ where $r_{0}$ and $A_{r_{0} / 2 k}(z)$ are such a constant and point as in Theorem 1.3. Then Theorems 1.2 and 1.3 show the existence of a positive constant $C$ satisfying (2.1) for any $u$ and $v$ in $\mathscr{I}$ and $k=1,2, \cdots$. Now, let us show (2.2). Fix $r$ with $0<r \leqq r_{0} / 2$, and denote by $B$ the ball $B(r, z)$. Let $u$ belong to $\mathscr{F}$. Choose an exhaustion $\left\{\Omega_{j}\right\}_{j=1}^{\infty}$ of $\Omega$ such that $B \cap \Omega \subset \Omega, \Subset M$ for any $j$. Let $u_{j}$ be the solution of the Dirichlet problem

$$
L u_{\jmath}=0 \text { in } \Omega_{j} \backslash \bar{B}, \quad u_{\jmath}=0 \text { on } \partial \Omega_{\jmath} \backslash B, \quad u_{\jmath}=u \text { on } \partial B \cap \Omega_{\jmath} .
$$

By the $\delta$-positivity, $u$, is increasing as $j \rightarrow \infty$ and converges to a positive solution $u^{\sim} \leqq u$. By the property (b), $u-u^{\sim}$ is a nonnegative solution of minimal growth at infinity of the one point compactification of $\Omega \backslash \bar{B}$. Thus Theorem 1.6 shows that $u=u^{\sim}$ in $\Omega \backslash \bar{B}$. This approximation of $u$ together with Theorem 1.5 yields (2.2). Hence, by Lemma 2.2, $\mathscr{F}$ consists of one solution. The proof of the claim is now complete.

The claim implies that any sequence $\left\{y_{k}\right\}_{k=1}^{\infty}$ converging to $z$ is a fundamental sequence and two such sequences are equivalent. Similarly, we obtain that the Martin kernel $K(x, z)$ is continuous in $z$ on $S$ (cf. [7, Corollary 3.2]). Since $\lim \sup _{x \rightarrow z} K(x, z)>0$ and

$$
\lim _{x \rightarrow z} K\left(x, y_{k}^{\prime}\right)=0 \text { uniformly with respect to } k=1,2, \cdots
$$


if $\left\{y_{k}^{\prime}\right\}_{k=1}^{\infty}$ is a sequence in $\Omega$ such that $z$ is not its accumulation point, we obtain that $K(x, z) \neq K(x, \omega)$ for any $\omega$ in $\Sigma$ with $\omega \neq z$. Finally, suppose that a positive solution $v$ satisfies $v(\cdot) \leqq K(\cdot, z)$ for some $z$ in $S$. Then, $v(x) / v\left(x_{0}\right)$ satisfies (a) and (b), and so $v(x)=v\left(x_{0}\right) K(x, z)$. Thus $K(\cdot, z)$ is a minimal positive solution. This completes the proof of the theorem.

Q.E.D.

The following theorem, an immediate consequence of Theorem 2.1, is worthy to mention.

Theorem 2.3. Let $\Omega$ be a relatively compact Lipshitz domain of $M$ with nonempty boundary. Suppose that $(L, \Omega)$ is subcritical. Then $\bar{\Omega}^{L}=\bar{\Omega}$ and $\Sigma=\sigma=\partial \Omega$.

Proof. By Theorem 2.1, $\bar{\Omega}$ is imbedded into $\bar{\Omega}^{L}, \partial \Omega \subset \sigma$, and $\{K(\cdot, z)\}_{z \in \partial \Omega}$ separates points of $\partial \Omega$. Since $\bar{\Omega}$ is compact, this implies that $\Sigma=\partial \Omega$. Hence $\Sigma=\sigma=\partial \Omega$.

Q.E.D.

Remark. In the special case where $M=R^{n}$ and $L$ is an operator of divergence form (i.e., $b^{i}=d^{i}=0$ and $c=0$ ), Theorem 2.3 was proved in [7] (see also $[12,9,39,17,38,35])$.

\section{§ 3. Tensor Product Decomposition}

In this section we study the structure of positive solutions of a second order elliptic equation $P u=0$ in a product domain, where $P$ is a sum of tensor products of operators. A main theorem of this section is Theorem 3.5, which will be proved in Section 4.

Let $P$ be an elliptic operator of the form

$$
P=L \otimes I+W \otimes A,
$$

where $L$ is an elliptic operator (1.1) on $M$ satisfying (i) (iii), $I$ is the identity operator on a connected orientable Riemannian manifold $N$ of dimension $m$ and class $C^{2}, W$ is a multiplication by a positive measurable function $W(x)$ such that $W(x)$ and $W(x)^{-1}$ are both locally bounded, $A$ is formally self-adjoint elliptic differential operator on $N$ given by

$$
A=-\sum_{i, j=1}^{m} G^{-1 / 2} \partial_{\jmath}\left(G^{1 / 2} A^{i j} \partial_{i}\right)+\sum_{i=1}^{m}\left(B^{i} \partial_{i}-G^{-1 / 2} \partial_{i} G^{1 / 2} B^{i}\right)+C,
$$

where $G=\operatorname{det}\left(G_{i j}\right)$ with $G_{i j}$ being the Riemannian metric on $N$, the operators $A$ and $P$ satisfy the conditions (i) (iii) below (1.1) with obvious modifications. Here $L \otimes I$ denotes the tensor product of $L$ and $I$. Let $\Omega$ be a domain of $M$, and $D$ a relatively compact Lipshitz domain of $N$ with nonempty boundary or a compact domain without boundary. We shall investigate the structure of 
positive solutions of the equation

$$
P u=0 \quad \text { in } \Omega \times D .
$$

Main results of this section are concerned with the Martin boundary and Martin kernel for $(P, \Omega \times D)$. The results to be given will be proved only in the case where $\partial D \neq \phi$, since the proof for the other case is similar to and simpler than the proof for the case $\partial D \neq \phi$.

Let $\lambda_{0}<\lambda_{1} \leqq \lambda_{2} \leqq \cdots$ be the eigenvalues repeated according to multiplicity of the Dirichlet realization $A_{D}^{\sim}$ of $A$ on $L_{2}(D)$. Let $\varphi_{j}(j=0,1, \cdots)$ be an eigenfunction associated with $\lambda_{j}$ such that $\left\{\varphi_{j}\right\}_{j=1}^{\infty}$ is a complete orthonormal system of $L_{2}(D)$. Recall that the smallest eigenvalue $\lambda_{0}$ is simple and the corresponding eigenfunction $\varphi_{0}$ is positive in $D$ (see, for example, Theorem 1.4 in Section 1.2).

We begin with the following criterion for the existence of a positive solution of (3.3). (As for the notion $\Gamma\left(L+\lambda_{0} W, \Omega\right)$, see (1.9).)

Theorem 3.1. There exists a positive solution of (3.3) if and only if $\Gamma\left(L+\lambda_{0} W, \Omega\right) \geqq 0$.

Proof. If $\Gamma\left(L+\lambda_{0} W, \Omega\right) \geqq 0$, then Theorem 1.5 implies the existence of a positive solution $\phi$ of $\left(L+\lambda_{0} W\right) \psi=0$ in $\Omega$. Put $u(x, y)=\psi(x) \varphi_{0}(y)$. Then $u$ is a positive solution of (3.3). Conversely, assume that there exists a positive solution of (3.3). Suppose that $\Gamma\left(L+\lambda_{0} W, \Omega\right)<0$. Then there exists a Lipshitz domain $E \Subset \Omega$ such that $\gamma \equiv \Gamma\left(L+\lambda_{0} W, E\right)<0$. By Theorem 1.4, choose a positive solution $\phi$ of

$$
\left(L+\lambda_{0} W-\gamma\right) \psi=0 \text { in } E \text { with } \phi=0 \text { on } \partial E .
$$

Put $v(x, y)=\psi(x) \varphi_{0}(y)$. Then, $(P-\gamma) v=0$ in $E \times D$ and $v=0$ on $\partial(E \times D)$. On the other hand, $(P-\gamma) u=-\gamma u>0$ in $E \times D$. Thus we have by Theorem 1.4 that $u=C v$ for some $C>0$. But $u>0$ on $\partial E \times D$, which is a contradiction

Q.E.D.

Now, assume that $\Gamma\left(L+\lambda_{0} W, \Omega\right) \geqq 0$. Let $\left\{\Omega_{k}\right\}_{k=1}^{\infty}$ be an exhaustion of $\Omega$ such that $\Omega_{k}$ is a relatively compact Lipshitz subdomain of $\Omega, \Omega_{k} \Subset \Omega_{k+1}$ for any $k$, and $\Omega_{k} \uparrow \Omega$ as $k \rightarrow \infty$. Let $G^{k}$ and $H_{0}^{k}, k=1,2, \cdots$, be the Green's functions for the Dirichlet realizations $P_{\tilde{\Omega}_{k} \times D}$ and $\left(L+\lambda_{0} W\right)_{\Omega_{k}}$, respectively.

Lemma 3.2. Let $K$ be a compact subset of $\Omega_{k}$, and $U$ an open set such that $K \subset U \Subset \Omega_{k}$. Then there exists a positive constant $C$ depending only on $K, U$, and the operator $P$ such that for all $l \geqq k$ and $\left(x, x^{\prime}, y, y^{\prime}\right) \in K \times\left(\Omega_{k} \backslash \bar{U}\right) \times D^{2}$

$$
C \leqq G^{l}\left(x, x^{\prime}, y, y^{\prime}\right) /\left[H_{0}^{l}\left(x, x^{\prime}\right) \varphi_{0}(y) \varphi_{0}\left(y^{\prime}\right)\right] \leqq C^{-1} .
$$

Proof. We denote by $u^{l}$ the function in (3.4). Regarding $G^{l}\left(x, x^{\prime}, y, y^{\prime}\right)$ 
and $H_{0}^{l}\left(x, x^{\prime}\right) \varphi_{0}(y) \varphi_{0}\left(y^{\prime}\right)$ as function of $(x, y)$, we see that both functions are positive solutions of the equation $P u=0$ in $U \times D$ which vanish continuously on $U \times \partial D$. Then, by Theorems 1.2 and 1.3 , there exist positive constants $C^{\prime}$ and $C^{\prime \prime}$ such that for all $(x, y)$ in $K \times D$

$$
C^{\prime} \leqq u^{l}\left(x, x^{\prime}, y, y^{\prime}\right) / u^{l}\left(x_{0}, x^{\prime}, y_{0}, y^{\prime}\right) \leqq C^{\prime \prime},
$$

where $\left(x_{0}, y_{0}\right)$ is a fixed point in $K \times D$. Applying the same argument as above to the functions $u^{l}\left(x_{0}, x^{\prime}, y_{0}, y^{\prime}\right)$ of $\left(x^{\prime}, y^{\prime}\right)$, we obtain that with another constant $C^{\prime}$ and $C^{\prime \prime}$

$$
C^{\prime} \leqq u^{l}\left(x, x^{\prime}, y, y^{\prime}\right) / u^{l}\left(x_{0}, x_{0}^{\prime}, y_{0}, y_{0}^{\prime}\right) \leqq C^{\prime \prime}
$$

for all $\left(x, x^{\prime}, y, y^{\prime}\right)$ in $K \times\left(\Omega_{k} \backslash \bar{U}\right) \times D^{2}$, where $\left(x_{0}^{\prime}, y_{0}^{\prime}\right)$ is a fixed point in $\left(\Omega_{k} \backslash \bar{U}\right) \times D$. Put

$$
h\left(x, x^{\prime}\right)=\iint_{D \times D} G^{l}\left(x, x^{\prime}, y, y^{\prime}\right) \varphi_{0}(y) \varphi_{0}\left(y^{\prime}\right) d y d y^{\prime} .
$$

For any $f(x)$ in $H_{0}^{1}\left(\Omega_{l}\right) \cap C^{0}\left(\bar{\Omega}_{l}\right)$ with $\left(L *+\lambda_{0} W\right) f \in C^{0}\left(\bar{\Omega}_{l}\right)$, we have (cf. [33]) that

$$
\begin{aligned}
\int_{\Omega} h(x, & \left.x^{\prime}\right)\left(L^{*}+\lambda_{0} W\right) f(x) d x \\
& =\int d y^{\prime} \varphi_{0}\left(y^{\prime}\right) \iint_{\Omega \times D} G^{l}\left(x, x^{\prime}, y, y^{\prime}\right) P^{*}\left[f(x) \varphi_{0}(y)\right] d x d y \\
& =\int_{D} f\left(x^{\prime}\right)\left[\varphi_{0}\left(y^{\prime}\right)\right]^{2} d y^{\prime}=f\left(x^{\prime}\right) .
\end{aligned}
$$

Thus we get

$$
\iint_{D \times D} G^{l}\left(x, x^{\prime}, y, y^{\prime}\right) \varphi_{0}(y) \varphi_{0}\left(y^{\prime}\right) d y d y^{\prime}=H_{0}^{l}\left(x, x^{\prime}\right)
$$

for any $\left(x, x^{\prime}\right)$ in $\Omega_{l}^{2} \backslash\left\{x=x^{\prime}\right\}$. Therecore, multiplying (3.5) by $\left[\varphi_{0}(y) \varphi_{0}\left(y^{\prime}\right)\right]^{2}$ and integrating it on $D \times D$, we have

$$
1 / C^{\prime \prime} \leqq u^{l}\left(x_{0}, x_{0}^{\prime}, y_{0}, y_{0}^{\prime}\right) \leqq 1 / C^{\prime} .
$$

This together with (3.5) yields (3.4).

Q.E.D.

A direct consequence of Lemma 3.2 is the following

Theorem 3.3. $(P, \Omega \times D)$ is critical (or subcritical) if and only if $\left(L+\lambda_{0} W, \Omega\right)$ is critical (or subcritical).

In the remainder of this section we assume that $\left(L+\lambda_{0} W, \Omega\right)$ is subcritical. Let $G$ and $H_{\jmath}, j=0,1, \cdots$, be the minimal Green's functions for $(P, \Omega \times D)$ and 
$\left(L+\lambda_{j} W, \Omega\right)$, respectively. Here we have used Theorem 1.7 and the assumption $W>0$.

Theorem 3.4. For any $\left(x, x^{\prime}, y, y^{\prime}\right)$ in $\left(\Omega^{2} \backslash\left\{x=x^{\prime}\right\}\right) \times D^{2}$

$$
G\left(x, x^{\prime}, y, y^{\prime}\right)=\sum_{j=0}^{\infty} H_{j}\left(x, x^{\prime}\right) \varphi_{j}(y) \varphi_{j}\left(y^{\prime}\right) .
$$

where the right hand side of (3.7) converges uniformly on $K \times(\Omega \backslash \bar{U}) \times D^{2}$ for any compact subset $K$ and an open set $U$ with $K \subset U \Subset \Omega$.

Proof. Let $H_{j}^{k}$ be the Green's function of the Dirichlet realization $\left(L+\lambda_{j} W\right) \tilde{\Omega}_{k}$ of $L+\lambda_{j} W$ on $L_{2}\left(\Omega_{k}\right)$. The same argument as in the proof of (3.6) shows that

$$
\int_{D} G^{k}\left(x, x^{\prime}, y, y^{\prime}\right) \varphi_{\jmath}(y) d y=H_{\jmath}^{k}\left(x, x^{\prime}\right) \varphi_{\jmath}\left(y^{\prime}\right)
$$

for any $\left(x, x^{\prime}, y^{\prime}\right)$ in $\left(\Omega_{k}^{2} \backslash\left\{x=x^{\prime}\right\}\right) \times D, k=1,2, \cdots, j=0,1, \cdots$. Letting $k \rightarrow \infty$ we obtain that

$$
\int_{D} G\left(x, x^{\prime}, y, y^{\prime}\right) \varphi_{\jmath}(y) d y=H_{\jmath}\left(x, x^{\prime}\right) \varphi_{\jmath}\left(y^{\prime}\right)
$$

for any $\left(x, x^{\prime}, y^{\prime}\right)$ in $\left(\Omega^{2} \backslash\left\{x=x^{\prime}\right\}\right) \times D$ and $j=0,1, \cdots$. Thus

$$
\sum_{\jmath=0}^{\infty}\left[H_{\jmath}\left(x, x^{\prime}\right) \varphi_{\jmath}\left(y^{\prime}\right)\right]^{2}=\int_{D} G\left(x, x^{\prime}, y, y^{\prime}\right)^{2} d y .
$$

Choose open sets $U_{1}$ and $U_{2}$ such that $K \subset U_{1} \Subset U_{2} \Subset U$. It follows from (3.6) that

$$
C \leqq G\left(x, x^{\prime}, y, y^{\prime}\right) /\left[H_{0}\left(x, x^{\prime}\right) \varphi_{0}(y) \varphi_{0}\left(y^{\prime}\right)\right] \leqq C^{-1}
$$

for any $\left(x, x^{\prime}, y, y^{\prime}\right)$ in $\bar{U}_{2} \times(\Omega \backslash \bar{U}) \times D^{2}$. This implies that $G$ is bounded on $U_{2} \times(\Omega \backslash \bar{U}) \times D^{2}$. Furthermore, $G$ as a function of $(x, y)$ satisfies the equation $P G=0$ in $U \times D$. Thus, by Théorème 7.1 of [33], there exist positive constants $\alpha$ and $C^{\prime}$ such that

$$
\left|G\left(x, x^{\prime}, y, y^{\prime}\right)-G\left(z, x^{\prime}, y, y^{\prime}\right)\right| \leqq C^{\prime}[\operatorname{dist}(x, z)]^{a}
$$

for any $(x, z)$ in $U_{1}^{2}$. This implies that

$$
\sum_{j=0}^{\infty}\left\{\left[H_{j}\left(x, x^{\prime}\right)-H_{j}\left(z, x^{\prime}\right)\right] \varphi_{\jmath}\left(y^{\prime}\right)\right\}^{2} \leqq C[\operatorname{dist}(x, z)]^{2 \alpha}
$$

with another constant $C$. From (3.9) and (3.11) we obtain that the series in the right hand side of (3.7) converges, as functions of $(x, y)$, in $L_{2}\left(U_{1} \times D\right)$ uniformly with respect to $\left(x^{\prime}, y^{\prime}\right)$ in $(\Omega \backslash \bar{U}) \times D$; furthermore, the right hand side is a solution of $P u=0$ in $U_{1} \times D$ which vanish continuously on $U_{1} \times \partial D$. 
Thus we obtain by Corollarire 5.2 of [33] and Theorem 1.3 that the series converges uniformly on $K \times(\Omega \backslash \bar{U}) \times D^{2}$. This implies that both sides of (3.7) are continuous on $\left(\Omega^{2} \backslash\left\{x=x^{\prime}\right\}\right) \times D^{2}$; which together with (3.8) shows (3.7).

Q.E.D.

Let $\Sigma_{0}, \sigma_{0}, K_{0}$ and $\bar{\Omega}^{0}$ be the Martin boundary, minimal Martin boundary, Martin kernel, and Martin compactification for $\left(L+\lambda_{0} W, \Omega\right)$ with reference point $x_{0} \in \Omega$, respectively. Denote by $\Sigma, \sigma, K$, and $\overline{\Omega \times D}^{P}$ those corresponding to $(P, \Omega \times D)$ with reference point $\left(x_{0}, y_{0}\right) \in \Omega \times D$. Note that Theorem 2.1 implies that

(3.12) $\Omega \times \partial D \subset \sigma$ and the relative topology of $\Omega \times \bar{D}$ in $\overline{\Omega \times D}^{P}$ is equal to that of $\Omega \times \bar{D}$ in $M \times N$.

In what follows, $A+B$ will stand for the disjoint union of two sets $A$ and $B$.

Theorem 3.5. Suppose that $\left(L+\lambda_{0} W, \Omega\right)$ is subcritical. Assume the following conditions $(\mathrm{P} . \mathrm{I}) \sim(\mathrm{P}$. III).

(P. I) For each $j=1,2, \cdots$ and $\xi$ in $\Sigma_{0}$, there exists the limit (which is denoted by $\left.K_{j}(x, \xi)\right)$

$$
\lim _{x^{\prime} \rightarrow \xi} H_{j}\left(x, x^{\prime}\right) / H_{0}\left(x_{0}, x^{\prime}\right), \quad x \in \Omega .
$$

(P. II) The functions $K_{j}(x, \xi)$ are continuous on $\Omega \times \Sigma_{0}$.

(P.III) There exist subsets $\Sigma_{0 \infty}$ and $\Sigma_{00}$ of $\Sigma_{0}$ such that $\Sigma_{0}=\Sigma_{0 \infty}+\Sigma_{00}$ and

$$
\begin{aligned}
& K_{j}(x, \xi)>0 \quad \text { on } \quad \Omega \times \Sigma_{0 \infty} \quad \text { for all } j=1,2, \cdots \\
& K_{j}(x, \xi)=0 \quad \text { on } \quad \Omega \times \Sigma_{00} \quad \text { for all } j=1,2, \cdots \text {. }
\end{aligned}
$$

Then the following conclusions (i) (iv) hold.

(i) With $d$ being an ideal point outside of $\bar{D}$,

$$
\Sigma=\Omega \times \partial D+\Sigma_{0 \infty} \times \bar{D}+\Sigma_{00} \times\{d\} .
$$

(ii.0) For each $(\xi, d)$ in $\Sigma_{00} \times\{d\}$, a subset $U$ of $\overline{\Omega \times D}^{P}$ is a neighborhood of $(\xi, d)$ if and only if there exists a neighborhood $V$ of $\xi$ in $\bar{\Omega}^{0}$ such that

$$
U \supset\left(V \cap \Sigma_{00}\right) \times\{d\}+\left(V \cap\left(\Omega+\Sigma_{0 \infty}\right)\right) \times \bar{D} .
$$

(ii. $\infty)$ For each $(\xi, \eta)$ in $\Sigma_{0 \infty} \times \bar{D}$, a subset $U$ of $\overline{\Omega \times D}^{P}$ is a neighborhood of $(\xi, \eta)$ if and only if $U \supset V \times W$ for a neighborhood $V$ of $\xi$ in $\bar{\Omega}^{0}$ and a neighborhood $W$ of $\eta$ in $\bar{D}$.

(iii.0) For each $\xi \in \Sigma_{00}$,

$$
K(x, \xi, y, d)=K_{0}(x, \xi) \varphi_{0}(y) / \varphi_{0}\left(y_{0}\right) .
$$


(iii. $\infty)$ For each $(\xi, \eta)$ in $\Sigma_{0 \infty} \times \bar{D}$,

$$
K(x, \xi, y, \eta)=\sum_{j=0}^{\infty} K_{j}(x, \xi) \varphi_{j}(y)\left[\varphi_{j} / \varphi_{0}\right](\eta) / C(\xi, \eta),
$$

where $\left[\varphi_{j} / \varphi_{0}\right]$ is a continuous extension of the function $\varphi_{j} / \varphi_{0}$ on $D$ to $\bar{D}$, and $C(\xi, \eta)$ is a positive constant defined by

$$
C(\xi, \eta)=\sum_{j=0}^{\infty} K_{j}\left(x_{0}, \xi\right) \varphi_{j}\left(y_{0}\right)\left[\varphi_{j} / \varphi_{0}\right](\eta)
$$

Furthermore, the series (3.16) converges uniformly on each compact subset of $\Omega \times \bar{D}$.

(iv.0) $\left(\Sigma_{00} \times\{d\}\right) \cap \sigma=\left(\Sigma_{00} \cap \sigma_{0}\right) \times\{d\}$.

(iv. $\infty)\left(\Sigma_{0 \infty} \times \bar{D}\right) \cap \sigma \supset\left(\Sigma_{0 \infty} \cap \sigma_{0}\right) \times \bar{D}$.

Remark 3.6. We obtain as in the proof of Lemma 4.1 below that if $\Omega$ is a Lipshitz domain, then $\partial \Omega \subset \Sigma_{0 \infty}$; more precisely, the limit (3.13) for any $\xi \in \partial \Omega$ and $j$ exists, and $K_{j}(x, \xi), j=1,2, \cdots$, are positive continuous functions on $\Omega \times \partial \Omega$.

Remark 3.7. Since $H_{j}\left(x, x^{\prime}\right) \leqq H_{1}\left(x, x^{\prime}\right)$ for any $j=2,3, \cdots$, we see that if $K_{1}(x, \xi)$ exists and is equal to zero, then $K_{\jmath}(x, \xi)=0$ for any $j=2,3, \ldots$. An interesting open problem is whether $K_{\jmath}(x, \xi)>0$ for any $j=2,3, \cdots$ if $K_{1}(x, \xi)$ $>0$. We shall see in Sections 5 and 6 that this holds if $\left(L+\lambda_{0} W, \Omega\right)$ can be reduced to the one dimensional case.

\section{$\S 4$. Proof of Theorem 3.5}

This section is devoted to the proof of Theorem 3.5. The theorem yields, among others, a method of constructing the Martin boundary for a second order elliptic operator which can be expressed as a sum of tensor products like (3.1) in a product of domains.

Lemma 4.1. For $j=1,2, \cdots, \varphi_{j} / \varphi_{0}$ in $D$ has Hölder continuous extension to $\bar{D}$.

Proof. We have only to treat the case where $\partial D \neq \phi$. For $\varepsilon>0$, put $D_{\varepsilon}=\{y \in D ; \operatorname{dist}(y, \partial D)<\varepsilon\}$. Choose $\varepsilon$ so small that there exists a positive solution $h$ of the equation $\left(A-\lambda_{j}\right) h=0$ in a neighborhood $U$ of $\bar{D}_{s}$ such that $\inf \left\{h(y) ; y \in \bar{D}_{\varepsilon}\right\}>0$. Put

$$
A_{h}=-\sum_{i, j=1}^{m} h^{-2} G^{-1 / 2} \partial_{j}\left(h^{2} G^{1 / 2} A^{i j} \partial_{i}\right), \quad \varphi_{l}^{h}=\varphi_{l} / h .
$$

Then 


$$
A_{h} \varphi_{\jmath}^{h}=0=A_{h} \varphi_{0}^{h}+\left(\lambda_{\jmath}-\lambda_{0}\right) \varphi_{0}^{h}=0 \quad \text { in } U \cap D .
$$

Let $\psi_{ \pm}$be the solution of the Dirichlet problem

$$
A_{h} u=0 \text { in } D_{\varepsilon} \text { and } u=\max \left( \pm \varphi_{\jmath}^{h}, 0\right) \text { on } \partial D_{\varepsilon} \text {. }
$$

Clearly, $\phi_{ \pm}>0$ in $D_{\varepsilon}$ and $\varphi_{j}^{h}=\psi_{+}-\psi_{-}$. Thus we have only to prove that $\psi_{ \pm} / \varphi_{0}^{h}$ are Hölder continuous on $\bar{D}_{\varepsilon}$. Put

$$
u(y, t)=\phi_{ \pm}(y) \text { and } v(y, t)=\varphi_{0}^{h}(y) \cos \left[\left(\lambda_{j}-\lambda_{0}\right)^{1 / 2} t\right],
$$

where $t$ is a real number. Set $\delta=\pi /\left[4\left(\lambda_{0}-\lambda_{0}\right)^{1 / 2}\right]$. Then $u$ and $v$ are positive solutions of the equation

$$
\left(A_{h}-\partial^{2} / \partial t^{2}\right) w=0 \text { in }(U \cap D) \times(-\delta, \delta), \quad w=0 \text { on } \partial D \times(-\delta, \delta) .
$$

Since $\left(A_{h}-\partial^{2} / \partial t^{2}\right)$ is elliptic, the argument in the proof of Theorem 7.9 of [17] together with Theorem 1.3 in Section 1 shows that

$$
u(y, t) / v(y, t)
$$

is Hölder continuous on $\bar{D}_{\varepsilon} \times[-\delta / 2, \delta / 2]$. Since $u(y, 0)=\phi_{ \pm}(y)$ and $v(y, 0)=$ $\varphi_{0}^{h}(y)$, this proves the lemma.

Q.E.D.

Recall that $K\left(x, x^{\prime}, y, y^{\prime}\right)=G\left(x, x^{\prime}, y, y^{\prime}\right) / G\left(x_{0}, x^{\prime}, y_{0}, y^{\prime}\right)$, where $G$ is the minimal Green's function for $(P, \Omega \times D)$ given by (3.7).

Lemma 4.2. (i) For any sequence $\left\{\left(x_{k}^{\prime}, y_{k}^{\prime}\right)\right\}_{k=1}^{\infty}$ in $\Omega \times \bar{D}$ with $x_{k}^{\prime} \rightarrow \xi \in \Sigma_{00}$ as $k \rightarrow \infty$,

$$
\lim _{k \rightarrow \infty} K\left(x, x_{k}^{\prime}, y, y_{k}^{\prime}\right)=K_{0}(x, \xi) \varphi_{0}(y) / \varphi_{0}\left(y_{0}\right) .
$$

(ii) For any sequence $\left\{\left(x_{k}^{\prime}, y_{k}^{\prime}\right)\right\}_{k=1}^{\infty}$ in $\Omega \times \bar{D}$ with $x_{k}^{\prime} \rightarrow \xi \in \Sigma_{0 \infty}$ and $y_{k}^{\prime} \rightarrow \eta \in \bar{D}$ as $k \rightarrow \infty$,

$$
\lim _{k \rightarrow \infty} K\left(x, x_{k}^{\prime}, y, y_{k}^{\prime}\right)=\sum_{j=0}^{\infty} K_{\jmath}(x, \xi) \varphi_{\jmath}(y)\left[\varphi_{j} / \varphi_{0}\right](\eta) C(\xi, \eta)^{-1}
$$

where $C(\xi, \eta)$ is a constant given by (3.17). Furthermore, the series in (4.7) converges uniformly on each compact subset of $\Omega \times \bar{D}$.

Proof. We shall show only (ii), since the proof of (i) is similar. Let

$$
J\left(x, x^{\prime}, y, y^{\prime}\right)=G\left(x, x^{\prime}, y, y^{\prime}\right) /\left[H_{0}\left(x_{0}, x^{\prime}\right) \varphi_{0}\left(y^{\prime}\right)\right] .
$$

Then, by (3.10), for any compact subset $K$ of $\Omega$ there exists a constant $C$ such that

$$
C \varphi_{0}(y) \leqq J\left(x, x_{k}^{\prime}, y, y_{k}^{\prime}\right) \leqq C^{-1} \varphi_{0}(y)
$$

fo: any $(x, y)$ in $K \times D$ and $k \gg 1$. Thus, for any subsequence of $\left\{\left(x_{k}^{\prime}, y_{k}^{\prime}\right)\right\}_{k}$ 
there exists its subsequence $\left\{\left(x_{k_{l}}^{\prime}, y_{k_{l}}^{\prime}\right)\right\}_{l}$ such that $J\left(x, x_{k_{l}}^{\prime}, y, y_{k_{l}}^{\prime}\right)$ converges as $l \rightarrow \infty$ to a positive solution $I(x, y)$ uniformly on $K \times D$. By Lebesgue's dominated convergence theorem, Theorem 3.4, and Lemma 4.1,

$$
\begin{aligned}
& \int_{D} I(x, y) \varphi_{J}(y) d y=\lim _{l \rightarrow \infty} \int_{D} J\left(x, x_{k_{l}}^{\prime}, y, y_{k_{l}}^{\prime}\right) \varphi_{\jmath}(y) d y \\
& \quad=\lim _{l \rightarrow \infty} H_{j}\left(x, x_{k_{l}}^{\prime}\right) \varphi_{\jmath}\left(y_{k_{l}}^{\prime}\right) /\left[H_{0}\left(x_{0}, x_{k_{l}}^{\prime}\right) \varphi_{0}\left(y_{k_{l}}^{\prime}\right)\right]=K_{\jmath}(x, \xi)\left[\varphi_{\jmath} / \varphi_{0}\right](\eta) .
\end{aligned}
$$

This implies the limit function $I(x, y)$ is determined uniquely by $(\xi, \eta)$. Hence

$$
J(x, \xi, y, \eta) \equiv \lim _{k \rightarrow \infty} J\left\{x, x_{k}^{\prime}, y, y_{k}^{\prime}\right)=\sum_{j=0}^{\infty} K_{\jmath}(x, \xi) \varphi_{\jmath}(y)\left[\varphi_{\jmath} / \varphi_{0}\right](\eta),
$$

from which (4.7) follows. The latter half of the lemma (ii) can be shown along the line given in the proof of Theorem 3.4.

Lemma 4.3. If a finite nonnegative Borel measure $\gamma$ on $\bar{D}$ satisfies, for some $\eta$ in $\bar{D}$,

$$
\left[\varphi_{J} / \varphi_{0}\right](\eta)=\int_{\bar{D}}\left[\varphi_{j} / \varphi_{0}\right](z) d \gamma_{z} \quad \text { for any } \quad j=0,1, \cdots
$$

then $\gamma$ is equal to the probability measure concentrated on the point $\eta$.

Proof. Consider the operator $P=-\partial^{2} / \partial x^{2}+A-\lambda_{0}$ in $(-1,1) \times D$. Then we see from the proof of Theorem 2.1 that the Martin compactification of $(-1,1) \times D$ with respect to $P$ is equal to $\overline{(-1,1) \times D}$, and the boundary points are all minimal. With the same notation as in Theorem 3.5 , we have

$$
K_{\jmath}(x, 1)=\lim _{x^{\prime} \rightarrow 1} H_{\jmath}\left(x, x^{\prime}\right) / H_{0}\left(x, x^{\prime}\right)>0
$$

for any $x$ in $(-1,1)$ and $j=1,2, \cdots$. Thus Lemma 4.2 implies that

$$
K(x, 1, y, \eta)=\sum_{j=0}^{\infty} K_{j}(x, 1) \varphi_{j}(y)\left[\varphi_{j} / \varphi_{0}\right](\eta) C(1, \eta)^{-1} .
$$

Thus, by the assumption of the lemma,

$$
K(x, 1, y, \eta)=\int_{\bar{D}} K(x, 1, y, z)[C(1, z) / C(1, \eta)] d r_{z} .
$$

Since $K(x, 1, y, \eta)$ is minimal, this implies the lemma.

Q.E.D.

Proof of Theorem 3.5. In view of Theorem 2.1 and Lemma 4.2, put

$$
K(x, X, y, Y)=\lim _{x^{\prime} \rightarrow X, y^{\prime} \rightarrow Y} K\left(x, x^{\prime}, y, y^{\prime}\right) \quad \text { for } \quad(X, Y) \in \Omega \times \partial D,
$$




$$
\begin{array}{ll}
K(x, \xi, y, \eta)=\lim _{x^{\prime} \rightarrow \xi, y^{\prime} \rightarrow \eta} K\left(x, x^{\prime}, y, y^{\prime}\right) & \text { for } \quad(\xi, \eta) \in \Sigma_{0 \infty} \times \bar{D}, \\
K(x, \xi, y, d)=\lim _{x^{\prime} \rightarrow \xi} K\left(x, x^{\prime}, y, y^{\prime}\right) & \text { for } \xi \in \Sigma_{00} .
\end{array}
$$

We claim that functions in the set

$$
\left\{K(x, u, y, v) ;(u, v) \in \Omega \times \partial D+\Sigma_{0 \infty} \times \bar{D}+\Sigma_{00} \times\{d\}\right\}
$$

are different each other. To this end it suffices to show that if $K(x, \xi, y, \eta)=$ $K\left(x, \xi^{\prime}, y, \eta^{\prime}\right)$, then $(\xi, \eta)=\left(\xi^{\prime}, \eta^{\prime}\right)$. Assume that $K(x, \xi, y, \eta)=K\left(x, \xi^{\prime}, y, \eta^{\prime}\right)$. Multiplying the both sides by $\varphi_{j}(y)$ and integrating them on $D$ with respect to $y$, we obtain that

$$
K_{j}(x, \xi)\left[\varphi_{j} / \varphi_{0}\right](\eta)=C^{\prime} K_{\jmath}\left(x, \xi^{\prime}\right)\left[\varphi_{j} / \varphi_{0}\right]\left(\eta^{\prime}\right), \quad j=0,1, \cdots,
$$

where $C^{\prime}=C(\xi, \eta) / C\left(\xi^{\prime}, \eta^{\prime}\right)$. This implies that $C^{\prime}=1, \xi=\xi^{\prime}$, and $\left[\varphi_{j} / \varphi_{0}\right](\eta)=$ $\left[\varphi_{j} / \varphi_{0}\right]\left(\eta^{\prime}\right)$ for all $j=0,1, \cdots$. Thus, by Lemma $4.3, \eta=\eta^{\prime}$. This proves the claim. On the other hand, any sequence $\left\{\left(x_{k}^{\prime}, y_{k}^{\prime}\right)\right\}_{k=1}^{\infty}$ without accumulation points in $\Omega \times D$ has a subsequence $\left\{\left(x_{k_{l}}^{\prime}, y_{k_{l}}^{\prime}\right)\right\}_{l=1}^{\infty}$ such that one of the following statements (a) $\sim$ (c) holds :

(a) The subsequence converges to a point in $\Omega \times \partial D$.

(b) It converges to a point in $\Sigma_{0 \infty} \times \bar{D}$.

(c) The sequence $\left\{x_{k_{l}}^{\prime}\right\}_{l}$ converges to a point in $\Sigma_{00}$.

This implies (i) and (iii).

In view of (3.12), $\Omega \times D+\Sigma$ is compact with respect to the topology $\tau$ induced by neighborhood systems described in (ii). Thus, in order to prove (ii), it suffices to show that the identity map from $(\Omega \times D+\Sigma, \tau)$ onto $\overline{\Omega \times D}^{P}$ is continuous. But this follows from the assumption (P. I $\sim(\mathrm{P} . \mathrm{III})$ and the formula

$$
K(x, X, y, Y)=\sum_{j=0}^{\infty}\left[H_{j}(x, X) / H_{0}\left(x_{0}, X\right)\right] \varphi_{j}(y)\left[\varphi_{j} / \varphi_{0}\right](Y) C(X, Y)^{-1}
$$

for any $(x, X, y, Y)$ in $\left(\Omega^{2} \backslash\left\{X=x\right.\right.$ or $\left.\left.x_{0}\right\}\right) \times D \times \partial D$, where $C(X, Y)$ is determined by $K\left(x_{0}, X, y_{0}, Y\right)=1$.

Let us show (iv. 0$)$. Assume that $K(x, \xi, y, d)$ is minimal. If a positive solution $\phi$ of the equation $\left(L+\lambda_{0} W\right) \psi=0$ in $\Omega$ satisfies $\psi(x) \leqq K_{0}(x, \xi)$ in $\Omega$, then

$$
\psi(x) \varphi_{0}(y) / \varphi_{0}\left(y_{0}\right) \leqq K(x, \xi, y, d) .
$$

This implies that $\phi(x) \varphi_{0}(y) / \varphi_{0}\left(y_{0}\right)=C K_{0}(x, \xi) \varphi_{0}(y) / \varphi_{0}\left(y_{0}\right)$ for some constant $C$. Thus $\phi(x)=C K_{0}(x, \xi)$, which shows that $K_{0}(\cdot, \xi)$ is minimal. Conversely, suppose that $K(x, \xi, y, d)$ is not minimal. Then there exist finite nonnegative Borel measures $\lambda$ on $\Omega \times \partial D, \mu$ on $\Sigma_{00}$ with $\mu(\{\xi\})=0$, and $\nu$ on $\Sigma_{0 \infty} \times \bar{D}$ such that 


$$
\begin{aligned}
K(x, \xi, y, d)= & \int_{\Omega \times \partial D} K(x, X, y, Y) d \lambda_{X, Y} \\
& +\int_{\Sigma_{00}} K(x, \zeta, y, d) d \mu_{\zeta}+\int_{\Sigma_{0 \infty \times} \times \bar{D}} K(x, \zeta, y, z) d \nu \zeta, z
\end{aligned}
$$

Choose an exhaustion $\left\{\Omega_{k}\right\}_{k=1}^{\infty}$ of $\Omega$ such that $\Omega_{k} \Subset \Omega_{k+1} \Subset \Omega$ for any $k$, and put

$$
u_{k}(x, y)=\int_{\Omega_{k} \times \partial D} K(x, X, y, Y) d \lambda_{X, Y} .
$$

Clearly, $u_{k}$ is of minimal growth at $\delta(\Omega \times D) \backslash\left(\Omega_{k+1} \times \partial D\right)$, where $\delta(\Omega \times D)$ is the boundary of $\Omega \times D$ in $\bar{M} \times \bar{D}$ with $\bar{M}$ being the one point compactification of $M$. On the other hand, since

$$
u_{k}(x, \xi) \leqq K_{0}(x, \xi) \varphi_{0}(y) / \varphi_{0}\left(y_{0}\right),
$$

$u_{k}$ is of minimal growth at $\Omega_{k+2} \times \partial D$. Thus $u_{k}$ is of minimal growth at $\delta(\Omega \times D)$, which together with Theorem 1.6 shows that $u_{k}=0$ for any $k$. This implies that $\lambda=0$. Similarly, $\nu=0$. The equation (4.15) now becomes

$$
K_{0}(x, \xi) \varphi_{0}(y) / \varphi_{0}\left(y_{0}\right)=\left(\int_{\Sigma_{00}} K_{0}(x, \zeta) d \mu_{\zeta}\right) \varphi_{0}(y) / \varphi_{0}\left(y_{0}\right),
$$

where $\mu(\{\xi\})=0$. This implies that $K_{0}(x, \xi)$ is not minimal. The proof of (iv. 0 ) is complete. It remains to prove (iv. $\infty$ ). Assuming that $K(x, \xi, y, \eta)$ is not minimal, we shall show that $K_{0}(x, \xi)$ is not minimal. We obtain in the same way as above that

$$
K(x, \xi, y, \eta)=\int_{\Sigma_{0 \infty} \times \bar{D}} K(x, \zeta, y, z) d \nu_{\zeta, 2}
$$

for some finite nonnegative Borel measure $\nu$ on $\Sigma_{0 \infty} \times \bar{D}$ with $\nu(\{\xi, \eta\})=0$. Multiply both sides of (4.16) by $\varphi_{j}(y)$ and integrate them on $D$ with respect to $y$. Then we have by Fubini's theorem that

$$
K_{\jmath}(x, \xi)\left[\varphi_{j} / \varphi_{0}\right](\eta)=\int_{\Sigma_{00 \infty} \times \bar{D}} K_{\jmath}(x, \zeta)\left[\varphi_{j} / \varphi_{0}\right](z) \frac{C(\xi, \eta)}{C(\zeta, z)} d \nu_{\zeta, z}
$$

for $j=0,1, \cdots$. The above equality for $j=0$ becomes

$$
K_{0}(x, \xi)=\int_{\Sigma_{00}} K_{0}(x, \zeta) d \lambda_{\zeta}
$$

where $\lambda$ is a finite measure on $\Sigma_{0 \infty}$ defined by

$$
\lambda(B)=\int_{B \times \bar{D}}[C(\xi, \eta) / C(\zeta, z)] d \nu \zeta, z
$$

for any Borel set $B$ of $\Sigma_{0 \infty}$. If $\lambda\left(\Sigma_{0 \infty} \backslash\{\xi\}\right)>0$, (4.18) already proves that $K_{0}(x, \xi)$ 
is not minimal. Therefore, suppose that $\lambda\left(\Sigma_{0 \infty} \backslash\{\xi\}\right)=0$. Then (4.17) becomes

$$
K_{j}(x, \xi)\left[\varphi_{j} / \varphi_{0}\right](\eta)=\int_{\bar{D}} K_{j}(x, \xi)\left[\varphi_{j} / \varphi_{0}\right](z) d \mu_{z},
$$

where $\mu$ is a measure on $\bar{D}$ defined by

$$
\mu(A)=\int_{(\xi) \times A}[C(\xi, \eta) / C(\zeta, z)] d \nu_{\zeta, z}
$$

for any Borel set $A$ of $\bar{D}$. Thus

$$
\left[\varphi_{j} / \varphi_{0}\right](\eta)=\int_{\bar{D}}\left[\varphi_{j} / \varphi_{0}\right](z) d \mu_{z}, \quad j=0,1, \cdots
$$

Since $\nu(\{\xi, \eta\})=0$, we have that $\mu(\{\eta\})=0$. Hence the above equalities contradict Lemma 4.3. This concludes that $K_{0}(x, \xi)$ is not minimal.

Q.E.D.

\section{$\S 5$. Schrödinger Equations in a Cone}

In order to illustrate the scope of the results in Section 3, we shall give applications of those results to equations in unbounded domains of $R^{n}$ in this and the next section.

Let $P$ be a Schrödinger operator

$$
P=-\Delta+V
$$

where $\Delta$ is the Laplace operator on $R^{n}$ and $V$ is a real-valued radial function in $L_{q, 10 c}\left(R^{n}\right)$, where $q>n / 2$ and $n \geqq 2$. Let $D$ be a Lipshitz domain in the unit sphere $S^{n-1}$ of $R^{n}$, and

$$
E=\left\{x \in R^{n} \backslash\{0\} ; x /|x| \subseteq D\right\} .
$$

In this section we investigate positive solutions of $P u=0$ in $E$. We should mention here that in the special case where $D=S^{n-1}$ the results to be given in this section are essentially the same as those given in [23, Section 3] (see also [26] and [14]). But we shall state the results without excluding those for the special case, which will be used in the next section.

We see that (5.1) can be rewritten as

$$
P=-r^{1-n} \partial / \partial r\left(r^{1-n} \partial / \partial r\right)+V(r)-r^{-2} \Lambda,
$$

where $\Lambda$ is the Laplace-Beltrami operator on $S^{n-1}$. Thus $P$ is of the form (3.1) with $M=R_{+} \equiv(0, \infty), N=S^{n-1}$,

$$
L=-r^{1-n} \partial / \partial r\left(r^{n-1} \partial / \partial r\right)+V(r), \quad A=-\Lambda, \text { and } W=r^{-2} .
$$

Let $\lambda_{0}<\lambda_{1} \leqq \lambda_{2} \leqq \cdots$ be the eigenvalues repeated according to multiplicity of the Dirichlet realization of $-\Lambda$ on $L_{2}(D)$, and $\varphi_{j}$ an eigenfunction associated with 
$\lambda_{j}$ chosen so that $\left(\varphi_{i}, \varphi_{j}\right)=\delta_{i j}$, where $\delta_{i j}=1$ if $i=j$ and $\delta_{i j}=0$ if $i \neq j$. Put

$$
\begin{aligned}
& \alpha_{j}=1-n / 2+\left[(1-n / 2)^{2}+\lambda_{j}\right]^{1 / 2}, \\
& \beta_{j}=1-n / 2-\left[(1-n / 2)^{2}+\lambda_{j}\right]^{1 / 2}, \\
& L_{j}=-r^{1-n} \partial / \partial r\left(r^{n-1} \partial / \partial r\right)+V(r)+\lambda_{j} r^{-2} .
\end{aligned}
$$

It is easily seen that for any $j=0,1, \cdots$ there exists a unique solution $g_{j}$ of the initial value problem

$$
\begin{aligned}
& L_{j} g_{j}=0 \quad \text { on } \quad R_{+}, \\
& g_{j}(r)=r^{\alpha_{j}}+O\left(r^{\alpha{ }^{+1}}\right) \quad \text { as } \quad r \rightarrow 0 .
\end{aligned}
$$

Theorem 5.1. There exists a positive solution of the equation $P u=0$ in $E$ if and only if $g_{0}>0$ on $R_{+}$.

Proof. In view of Theorems 3.1 and 1.5, it suffices to show that $g_{0}>0$ if the equation $(5.5)_{0}$ has a positive solution $h$. Since $g_{0}(r)>0$ near 0 , it is obvious that if $h$ is a constant multiple of $g_{0}$, then $g_{0}>0$ on $R_{+}$. Thus assume that $h$ is linearly independent of $g_{0}$. Since (5.5) $)_{0}$ has a solution $f$ such that

$$
f(r)=[1+o(1)] \max \left(r^{\beta_{0}}, \log (1 / r)\right) \quad \text { as } r \rightarrow 0,
$$

we obtain by $(5.6)_{0}$ that

$$
g_{0}(r)=C h(r) \int_{0}^{r} t^{1-n} h^{-2}(t) d t
$$

for some constant $C$. This proves that $g_{0}>0$.

Q.E.D.

Theorem 5.2. (i) (P,E) is subcritical if and only if for each $\delta>0$

$$
\int_{\delta}^{\infty} t^{1-n}\left(g_{0}(t)\right)^{-2} d t<\infty
$$

(ii) $(P, E)$ is critical if and only if $g_{0}>0$ and the integral in (5.7) deverges.

Proof. We see that

$$
\begin{aligned}
& r^{(n-1) / 2} L_{0} r^{-(n-1) / 2} \\
& =-\partial^{2} / \partial r^{2}+V(r)+\left[\lambda_{0}+\frac{(n-1)(n-3)}{4}\right] r^{-2} \equiv L_{0}^{\prime} .
\end{aligned}
$$

Thus, by Theorem 3.3, $(P, E)$ is critical if and only if $\left(L_{0}^{\prime}, R_{+}\right)$is critical. But Theorem A.5 in [23, Appendix] implies that $\left(L_{0}^{\prime}, R_{+}\right)$is critical if and only if $g_{0}>0$ and the integral in (5.7) diverges. This proves (ii); and also (i) because if $g_{0}(s)=0$ for some $s>0$, then (5.7) does not hold.

Q.E.D. 
Theorem 5.3. Assume (5.7). Then the minimal Green's function G for $(P, E)$ is given by

$$
G\left(r, r^{\prime}, \omega, \omega^{\prime}\right)=\sum_{j=0}^{\infty} g_{j}(r) f_{j}\left(r^{\prime}\right) \varphi_{j}(\omega) \varphi_{j}\left(\omega^{\prime}\right)
$$

for $0<r \leqq r^{\prime}<\infty$ and $\left(\omega, \omega^{\prime}\right) \in D^{2}$, where

$$
f_{j}\left(r^{\prime}\right)=g_{j}\left(r^{\prime}\right) \int_{r^{\prime}}^{\infty} t^{1-n} g_{j}(t)^{-2} d t
$$

Furthermore, $G\left(r, r^{\prime}, \omega, \omega^{\prime}\right)=G\left(r^{\prime}, r, \omega, \omega^{\prime}\right)$ for $0<r^{\prime} \leqq r<\infty$.

Proof. Denote by $H_{j}\left(r, r^{\prime}\right)$ the minimal Green's function for $\left(L_{\jmath}, R_{+}\right)$. Noting that we take $r^{n-1} d r$ as the volume element of $R_{+}$, we obtain by elementary calculations that

$$
\begin{aligned}
& H_{j}\left(r, r^{\prime}\right)=g_{j}(r) f_{j}\left(r^{\prime}\right) \quad \text { for } \quad 0<r \leqq r^{\prime}<\infty, \\
& =f_{j}(r) g_{j}\left(r^{\prime}\right) \text { for } 0<r^{\prime} \leqq r<\infty .
\end{aligned}
$$

This together with Theorem 3.4 proves the theorem.

Q.E.D.

In the remainder of this section we assume (5.7). Let $\Sigma_{0}, \sigma_{0}, \bar{R}_{+}^{0}$ and $K_{0}$ be the Martin boundary, minimal Martin boundary, Martin compactification of $R_{+}$, and Martin kernel for $\left(L_{0}, R_{+}\right)$with reference point 1 , respectively. Then we obtain from Theorem A.7 of [23] that

$$
\begin{array}{cl}
\Sigma_{0}=\sigma_{0}=\{0, \infty\}, & \bar{R}_{+}^{0}=[0, \infty], \\
K_{0}(r, 0)=f_{0}(r) / f_{0}(1), & K_{0}(r, \infty)=g_{0}(r) / g_{0}(1) .
\end{array}
$$

Denote by $\Sigma, \sigma, \bar{E}^{P}$ and $K$ those corresponding to $(P, E)$ with reference point $\left(1, \omega_{0}\right) \in R_{+} \times D$, respectively. In the following theorems, we shall use the notation $K(r, \omega ; * \eta)$ instead of $K(r, *, \omega, \eta)$ with $*=0$ or $\infty$.

Theorem 5.4. Assume that

$$
\int_{1}^{\infty} t^{n-3} g_{0}(t)^{2}\left(\int_{t}^{\infty} s^{1-n} g_{0}(s)^{-2} d s\right) d t=\infty .
$$

Then, with $0 d$ and $\infty$ d being ideal points at zero and infinity, respectively,

$$
\Sigma=\sigma=R_{+} \times \partial D+\{0 d, \infty d\} ;
$$

$\bar{E}^{P}$ is, as a topological space, the closure of $E$ in the one point compactification of $R^{n}$; and

$$
\begin{aligned}
& K(r, \omega ; 0 d)=f_{0}(r) \varphi_{0}(\omega) /\left[f_{0}(1) \varphi_{0}\left(\omega_{0}\right)\right], \\
& K(r, \omega ; \infty d)=g_{0}(r) \varphi_{0}(\omega) /\left[g_{0}(1) \varphi_{0}\left(\omega_{0}\right)\right] .
\end{aligned}
$$


Proof. We have by (5.6) and (5.11) that for any $j=1,2, \cdots$

$$
\lim _{r^{\prime} \rightarrow 0} \frac{H_{j}\left(r, r^{\prime}\right)}{H_{0}\left(1, r^{\prime}\right)}=\left(\lim _{r^{\prime} \rightarrow 0} \frac{g_{j}\left(r^{\prime}\right)}{g_{0}\left(r^{\prime}\right)}\right) \frac{f_{j}(r)}{f_{0}(1)}=0 \text {. }
$$

By (5.11),

$$
\lim _{r^{\prime} \rightarrow \infty} \frac{H_{j}\left(r, r^{\prime}\right)}{H_{0}\left(1, r^{\prime}\right)}=\left(\lim _{r^{\prime} \rightarrow \infty} \frac{f_{j}\left(r^{\prime}\right)}{f_{0}\left(r^{\prime}\right)}\right) \frac{g_{j}(r)}{g_{0}(1)} .
$$

But elementary calculations show that (5.14) holds if and only if

$$
\lim _{s \rightarrow \infty} f_{j}(s) / f_{0}(s)=0 \quad \text { for all } j=1,2, \cdots
$$

(cf. proofs of Theorems 3.2 and 3.3 in [23]). With the same notation as in Theorem 3.5, (5.17), (5.18) and (5.19) show that $\Sigma_{00}=\{0, \infty\}=\Sigma_{0}$. Hence Theorem 3.5 together with (5.12) and (5.13) yield the theorem. Q.E.D.

Theorem 5.5. Assume that

$$
\int_{1}^{\infty} t^{n-3} g_{0}(t)^{2}\left(\int_{t}^{\infty} s^{1-n} g_{0}(s)^{-2} d s\right) d t<\infty
$$

Then

$$
\Sigma=\sigma=R_{+} \times \partial D+\{0 d\}+\{\infty \xi ; \xi \in \bar{D}\} ;
$$

$\bar{E}^{P}$ is, as a topological space, the closure of $E$ in the compactification $R^{n}+\left\{\infty \omega ; \omega \in S^{n-1}\right\}$ of $R^{n}$ obtained by attaching the unit sphere at infinity to $R^{n}$; and there hold the formulas (5.15) and

$$
K(r, \omega ; \infty \xi)=\sum_{j=0}^{\infty} a_{j} g_{j}(r) \varphi_{j}(\omega)\left[\varphi_{j} / \varphi_{0}\right](\xi) C(\infty \xi)^{-1},
$$

where $a$, are positive constants defined by

$$
a_{j}=\lim _{r \rightarrow \infty} g_{0}(r) / g_{j}(r)
$$

and $C(\infty \xi)=\sum_{\jmath=0}^{\infty} a_{j} g_{\jmath}(1) \varphi_{\jmath}\left(\omega_{0}\right)\left[\varphi_{j} / \varphi_{0}\right](\xi)$.

Proof. We obtain along the line given in Lemma 3.7 of [23] that if (5.20) holds, then the limits $(5.22)$ exist and are positive. With $h_{j}=g_{0} / g_{j}$ we have

$$
\frac{f_{j}(r)}{f_{0}(r)}=\frac{\int_{r}^{\infty} t^{1-n} g_{0}(t)^{-2} h_{j}(t)^{2} d t}{h_{j}(r) \int_{r}^{\infty} t^{1-n} g_{0}(t)^{-2} d t} .
$$

Thus $f_{j}(r) / f_{0}(r) \rightarrow a_{j}$ as $r \rightarrow \infty$, which together with (5.18) implies that

$$
\lim _{r^{\prime} \rightarrow \infty} H_{j}\left(r, r^{\prime}\right) / H_{0}\left(1, r^{\prime}\right)=a_{j} g_{j}(r) / g_{0}(1) .
$$


Theorem 3.5, (5.17) and (5.23) imply the theorem.

Q.E.D.

Remark 5.6. The results of this section can be extended to Schrödinger operators with principally radially symmetric potentials along the line given in [23, Section 5].

We conclude this section with a remark concerning relevant results.

Remark 5.7. The results of this section considerably extend those in [23, Section 6], where the half space case was treated (see also [36]). In the case where $P=-\Delta$ and $E$ is a cone with nonempty boundary, the solution $g_{0}$ of the initial value problem (5.5) $)_{0}$ and $(5.6)_{0}$ is given by $g_{0}(r)=r^{\alpha_{0}}$ with $\alpha_{0}>0$; and so (5.7) and (5.14) hold. Thus Theorem 5.4 implies that there exists a unique positive solution of $-\Delta u=0$ in $E$ with $u=0$ on $\partial E$ and $u\left(1, \omega_{0}\right)=1$. This kind of uniqueness theorem for uniformly elliptic equations of divergence form was given in [19]. We shall show in Appendix an extension of it as an application of the comparison theorem (Theorem 1.3 in Section 1) and a criterion of uniqueness (Lemma 2.2 in Section 2).

\section{§6. Equations in the Product of a Cone and a Bounded Domain}

In this section we study positive solutions of an elliptic equation in the product of a cone in $R^{n}$ and a bounded Lipshitz domain in $R^{m}$ by applying the results in Sections 3 and 5 .

Let $P$ be an elliptic operator on $R_{x}^{n} \times R_{y}^{m}(n \geqq 2$ and $m \geqq 1)$ of the form

$$
P=-\Delta_{x}+V(x)+W(x)\left[-\Delta_{y}+U(y)\right],
$$

where $V$ and $W$ are real-valued measurable radial functions on $R^{n}$ such that $V \in L_{q, \text { loc }}\left(R^{n}\right)$ for some $q>(m+n) / 2, W>0, W$ and $W^{-1}$ are both locally bounded, and $U$ is a real-valued function in $L_{r, 10 c}\left(R^{m}\right), r>(m+n) / 2$. Let $D$ be a Lipshitz domain with nonempty boundary in $S^{n-1}$, or $D=S^{n-1}$; and

$$
\begin{aligned}
E & =\left\{x \in R^{n} \backslash\{0\} ; x /|x| \in D\right\} & & \text { if } \partial D \neq \phi, \\
& =R^{n} & & \text { if } D=S^{n-1} .
\end{aligned}
$$

Let $F$ be a bounded Lipshitz domain in $R^{m}$. In this section we investigate positive solutions of the equation

$$
P u=0 \quad \text { in } E \times F .
$$

With $L=-\Delta_{x}+V$ and $A=-\Delta_{y}+U, P$ is rewritten as $P=L \otimes I+W \otimes A$; and $L$ is also rewritten as (5.2). Let $\left\{\lambda_{j}\right\}_{j=0}^{\infty}$ and $\left\{\varphi_{j}\right\}_{j=0}^{\infty}$ be the eigenvalues and eigenfunctions of $-\Lambda_{\bar{D}}$, respectively, as in Section 5 . Denote by $\left\{\mu_{k}\right\}_{k=0}^{\infty}$ and $\left\{\phi_{k}\right\}_{k=0}^{\infty}$ those corresponding to the Dirichlet realization $A_{F}^{\sim}$ on $L_{2}(F)$ of the 
operator $A$. Let $\alpha_{j}, \beta_{j}$ and $L_{j}(j=0,1, \cdots)$ be the constants and the operators defined by (5.3) and (5.4), respectively. Let $g_{j k}(j, k=0,1,2, \cdots)$ be the solution of the initial value problem

$$
\begin{aligned}
& \left(L_{j}+\mu_{k} W\right) g_{j k}=0 \quad \text { on } \quad R_{+}, \\
& g_{j k}(r)=r^{\alpha_{j}+o\left(r^{\alpha^{+1}}\right)} \quad \text { as } \quad r \rightarrow 0 .
\end{aligned}
$$

We write $\rho=g_{00}$.

Theorem 6.1. There exists a positive solution of (6.2) if and only if $\rho>0$ on $R_{+}$.

Proof. We treat only the case where $E=R^{n}$. Theorems 3.1 and $5.1 \mathrm{imply}$ that $\rho>0$ if and only if the equation $L^{\prime} u \equiv\left(L+\mu_{0} W\right) u=0$ in $R^{n} \backslash\{0\}$ has a positive solution $u$. Note that $u=C g+h$ in a sufficiently small punctured ball $B \backslash\{0\}$, where $g$ is the Green's function for $\left(L^{\prime}\right)_{B}^{\sim}$ and $h$ is a positive solution of $L^{\prime} h=0$ in $B$. Thus $u$ is equal to either a positive (not necessarily minimal) Green's function on $R^{n}$ with pole at the origin or a positive solution on $R^{n}$. Hence the existence of a positive solution in $R^{n} \backslash\{0\}$ is equivalent to that of a positive solution in $R^{n}$.

Q.E.D.

Theorem 6.2. (i) $(P, E \times F)$ is subcritical if and only if for each $\delta>0$

$$
\int_{\delta}^{\infty} t^{1-n} \rho(t)^{-2} d t<\infty .
$$

(ii) $(P, E \times F)$ is critical if and only if $\rho>0$ and the integral in (6.5) diverges.

Proof. We treat only the case where $E=R^{n}$. Since the minimal Green's function for $\left(L+\mu_{0} W, R^{n} \backslash\{0\}\right)$ is, if it exists, equal to the restriction to $R^{n} \backslash\{0\}$ of that for $\left(L+\mu_{0} W, R^{n}\right)$, the theorem follows from Theorems 3.3 and 5.2.

Q.E.D.

In the remainder of this section we assume (6.5).

Theorem 6.3. The minimal Green's function $G$ for $(P, E \times F)$ is given by

$$
G\left(x, x^{\prime}, y, y^{\prime}\right)=\sum_{k=0}^{\infty} H_{k}\left(x, x^{\prime}\right) \psi_{k}(y) \psi_{k}\left(y^{\prime}\right)
$$

for any $\left(x, x^{\prime}, y, y^{\prime}\right) \in\left(E^{2} \backslash\left\{x=x^{\prime}\right\}\right) \times F^{2}$, where

$$
H_{k}\left(x, x^{\prime}\right)=\sum_{j=0}^{\infty} g_{j k}(|x|) f_{j k}\left(\left|x^{\prime}\right|\right) \varphi_{\jmath}(x /|x|) \varphi_{\jmath}\left(x^{\prime} /\left|x^{\prime}\right|\right)
$$

for $\left(x, x^{\prime}\right)$ with $|x| \leqq\left|x^{\prime}\right|$, and $H_{k}\left(x, x^{\prime}\right)=H_{k}\left(x^{\prime}, x\right)$ for $\left|x^{\prime}\right| \leqq|x|$. Here 


$$
f_{j k}(s)=g_{j k}(s) \int_{s}^{\infty} t^{1-n}\left[g_{j k}(t)\right]^{-2} d t
$$

Proof. Theorems 3.4 and 5.3 yield the theorem.

Q.E.D.

Let $R^{n}+\{\infty\}$ be the one point compactification of $R^{n}$, and $R^{n}+\infty S^{n-1}$ the compactification of $R^{n}$ obtained by attaching the unit sphere at infinity. Denote by $\Sigma, \sigma, \overline{E \times F}^{P}$ and $K$ the Martin boundary, minimal Martin boundary, Martin compactification of $E \times F$, and Martin kernel for $(P, E \times F)$ with reference point $\left(x_{0}, y_{0}\right)$ in $D \times F$, respectively. Put

$$
\begin{aligned}
& I_{1}=\int_{1}^{\infty} t^{n-3} \rho(t)^{2}\left(\int_{t}^{\infty} s^{1-n} \rho(s)^{-2} d s\right) d t \\
& I_{2}=\int_{1}^{\infty} W(t) t^{n-1} \rho(t)^{2}\left(\int_{t}^{\infty} s^{1-n} \rho(s)^{-2} d s\right) d t
\end{aligned}
$$

Then we have the following theorems.

Theorem 6.4. Assume that $I_{1}=I_{2}=\infty$. Then

$$
\Sigma=\sigma=\partial(E \times F)+\{(\infty d, f)\},
$$

where $f$ is an ideal point outside of $\bar{F} ; \overline{E \times F}^{P}$ is, as a topological space, the closure of $E \times F$ in the one point compactification of $R^{n} \times \bar{F}$; and

$$
K(x, \infty d, y, f)=g_{00}(|x|) \varphi_{0}(x /|x|) \psi_{0}(y)\left[g_{00}(1) \varphi_{0}\left(x_{0}\right) \psi_{0}\left(y_{0}\right)\right]^{-1} .
$$

Theorem 6.5. Assume that $I_{1}=\infty$ and $I_{2}<\infty$. Then

$$
\Sigma=\sigma=\partial(E \times F)+\{(\infty d, \eta) ; \eta \in \bar{F}\} ;
$$

$\overline{E \times F}^{P}$ is, as a topological space, the closure of $E \times F$ in $\left(R^{n}+\{\infty\}\right) \times \bar{F}$; and

(6.14) $K(x, \infty d, y, \eta)=\sum_{k=0}^{\infty} a_{0 k} g_{0 k}(|x|) \varphi_{0}(x /|x|) \psi_{k}(y)\left[\psi_{k} / \psi_{0}\right](\eta) / C(\infty d, \eta)$, where $a_{0 k}$ are positive constants defined by

$$
a_{0 k}=\lim _{r \rightarrow \infty} g_{00}(r) / g_{0 k}(r),
$$

and $C(\infty d, \eta)$ is a constant chosen so that $K\left(x_{0}, \infty d, y_{0}, \eta\right)=1$.

Theorem 6.6. Assume that $I_{1}<\infty$ and $I_{2}=\infty$. Then

$$
\Sigma=\sigma=\partial(E \times F)+\{(\infty \xi, f) ; \xi \in \bar{D}\} ;
$$

a subset of $\overline{E \times F}^{P}$ is a neighborhood of $(\infty \xi, f)$ if and only if it contains a set of the form 


$$
(N \cap \infty \bar{D}) \times\{f\}+(N \cap \bar{E}) \times \bar{F}
$$

with $N$ being a neighborhood of $\infty \xi$ in $R^{n}+\infty S^{n-1}$; and

$$
K(x, \infty \xi, y, f)=\sum_{j=0}^{\infty} a_{j 0} g_{j 0}(|x|) \varphi_{j}(x /|x|)\left[\varphi_{j} / \varphi_{0}\right](\xi) \psi_{0}(y) / C(\infty \xi, f),
$$

where $a_{j 0}$ are positive constants defined by

$$
a_{j 0}=\lim _{r \rightarrow \infty} g_{00}(r) / g_{j 0}(r),
$$

and $C(\infty \xi, f)$ is a constant determined so that $K\left(x_{0}, \infty \xi, y_{0}, f\right)=1$.

Theorem 6.7. Assume that $I_{1}<\infty$ and $I_{2}<\infty$. Then

$$
\Sigma=\sigma=\partial(E \times F)+\{(\infty \xi, \eta) ; \xi \in \bar{D}, \eta \in \bar{F}\} ;
$$

$\overline{E \times F}^{P}$ is, as a topological space, the closure of $E \times F$ in $\left(R^{n}+\infty S^{n-1}\right) \times \bar{F}$; and

$$
\begin{aligned}
K(x, \infty \xi, y, \eta)= & \sum_{k=0}^{\infty} \sum_{j=0}^{\infty} a_{j k} g_{j k}(|x|) \varphi_{\jmath}(x /|x|)\left[\varphi_{j} / \varphi_{0}\right](\xi) \\
& \times \varphi_{j}(y)\left[\psi_{j} / \phi_{0}\right](\eta) / C(\infty \xi, \eta),
\end{aligned}
$$

where $a_{j k}$ are positive constants defined by

$$
a_{j k}=\lim _{r \rightarrow \infty} g_{00}(r) / g_{j k}(r),
$$

and $C(\infty \xi, \eta)$ is a constant chosen so that $K\left(x_{0}, \infty \xi, y_{0}, \eta\right)=1$.

We need several lemmas in order to prove Theorems 6.4 6.7.

Lemma 6.8. Let $h_{k}(r)=g_{0 k}(r) / g_{00}(r), k=1,2, \ldots$. Then one has:

(i) $h_{k}$ are differentiable strictly increasing positive functions on $R_{+}$.

(ii) The following conditions (a), (b) and (c) are equivalent each other:

(a) $I_{2}=\infty$; (b) $\lim _{r \rightarrow \infty} h_{1}(r)=\infty$; and (c) $\lim _{r \rightarrow \infty} h_{k}(r)=\infty$ for any $k=1,2, \cdots$.

(iii) The following conditions $\left(\mathrm{a}^{\prime}\right),\left(\mathrm{b}^{\prime}\right)$ and $\left(\mathrm{c}^{\prime}\right)$ are equivalent each other:

(a') $I_{2}<\infty$; ( $\left.\mathrm{b}^{\prime}\right) \lim _{r \rightarrow \infty} h_{1}(r)<\infty$; and $\left(\mathrm{c}^{\prime}\right) \lim _{r \rightarrow \infty} h_{k}(r)<\infty$ for any $k=1,2, \cdots$.

(iv) $\lim _{r \rightarrow \infty} f_{0 k}(r) / f_{00}(r)=\left[\lim _{r \rightarrow \infty} h_{k}(r)\right]^{-1}$ for any $k=1,2, \cdots$.

Proof. We have from (6.3) and (6.4) that when $\gamma \equiv \alpha_{0}-\beta_{0}>0$,

$$
g_{0 k}(r) r^{-\alpha_{0}}=1+\int_{0}^{r} \frac{1-(t / r)^{r}}{\gamma} t\left(V+\mu_{k} W\right)(t)\left(g_{0 k}(t) t^{-\alpha_{0}}\right) d t ;
$$

and when $\alpha_{0}-\beta_{0}=0$ (i.e., $\alpha_{0}=\beta_{0}=0$ and $n=2$ ),

$$
g_{0 k}(r)=1+\int_{0}^{r}[\log (r / t)] t\left(V+\mu_{k} W\right)(t) g_{0 k}(t) d t
$$


Thus, $h_{k}(r) \rightarrow 1$ as $r \rightarrow 0$. Furthermore,

$$
\begin{aligned}
& {\left[g_{00}(r)\right]^{2} h_{k}^{\prime}(r)} \\
& =\int_{0}^{r}(t / r)^{\gamma+1}\left(\left(V+\mu_{k} W\right)(t) g_{0 k}(t)-\left(V+\mu_{0} W\right)(t) g_{00}(t)\right) t^{-\alpha_{0}} d t .
\end{aligned}
$$

This implies that $h_{k}^{\prime}(r) \rightarrow 0$ as $r \rightarrow 0$. On the other hand, with $\rho=g_{00}$ we have that

$$
\left[\rho^{2}(r) r^{n-1} h_{k}^{\prime}(r)\right]^{\prime}=r^{n-1} \rho^{2}(r)\left(\mu_{k}-\mu_{0}\right) W(r) h_{k}(r) .
$$

Therefore we get

$$
\begin{aligned}
& h_{k}(r)=1+\left(\mu_{k}-\mu_{0}\right) \int_{0}^{r} F(r, t) h_{k}(t) d t, \\
& F(r, t)=W(t) \int_{t}^{r}(t / s)^{n-1}[\rho(t) / \rho(s)]^{2} d s .
\end{aligned}
$$

This implies (i).

Let us show (ii) and (iii). Suppose that $I_{2}=\infty$. Since $h_{k} \geqq 1$ we have from (6.24) that

$$
h_{k}(r) \geqq 1+\left(\mu_{k}-\mu_{0}\right) \int_{0}^{r} F(r, t) d t .
$$

This together with the monotone convergence theorem implies that

$$
\lim _{r \rightarrow \infty} h_{k}(r) \geqq 1+\left(\mu_{k}-\mu_{0}\right) I_{2}=\infty
$$

for any $k=1,2, \cdots$. Conversely, suppose that $I_{2}<\infty$. With $k$ fixed, choose $R$ so large that

$$
\left(\mu_{k}-\mu_{0}\right) \int_{R}^{\infty} F(\infty, t) d t<1 / 2,
$$

where $F(\infty, t)=\lim _{r \rightarrow \infty} F(r, t)$. Then we have that for any $r>R$

$$
h_{k}(r) \leqq 1+\left(\mu_{k}-\mu_{0}\right) \int_{0}^{R} F(\infty, t) h_{k}(t) d t+h_{k}(r) / 2 .
$$

This implies that $\lim _{r \rightarrow \infty} h_{k}(r)<\infty$. Hence we get (ii) and (iii).

It remains to show (iv). By (6.8),

$$
\frac{f_{0 k}(r)}{f_{00}(r)}=\frac{h_{k}(r) \int_{r}^{\infty} h_{k}(t)^{-2} t^{1-n} \rho(t)^{-2} d t}{\int_{r}^{\infty} t^{1-n} \rho(t)^{-2} d t} .
$$

Suppose that $h_{k}(r) \rightarrow \infty$ as $r \rightarrow \infty$. Since $h_{k}$ is increasing, $f_{0 k}(r) / f_{00}(r) \leqq h_{k}(r)^{-1}$; which implies that

$$
f_{0 k}(r) / f_{00}(r) \longrightarrow 0 \quad \text { as } \quad r \rightarrow \infty
$$


On the other hand, if $\lim _{r \rightarrow \infty} h_{k}(r)<\infty$, then (6.25) clearly implies (iv). Q.E.D.

Lemma 6.9. Let $h_{j k}=g_{j_{k}} / g_{0 k}, j=1,2, \cdots$ and $k=0,1, \cdots$. Put

$$
J_{k}=\int_{1}^{\infty} t^{n-3}\left[g_{0 k}(t)\right]^{2}\left(\int_{t}^{\infty} s^{1-n}\left[g_{0 k}(s)\right]^{-2} d s\right) d t .
$$

Then one has:

(i) $h_{\jmath_{k}}$ are differentiable strictly increasing positive functions on $R_{+}$.

(ii) With $k$ fixed, the following conditions (a), (b) and (c) are equivalent each other: (a) $J_{k}=\infty$; (b) $\lim _{r \rightarrow \infty} h_{1 k}(r)=\infty$; and (c) $\lim _{r \rightarrow \infty} h_{j k}(r)=\infty$ for any $j=1,2, \cdots$.

(iii) With $k$ fixed, the following conditions $\left(\mathrm{a}^{\prime}\right),\left(\mathrm{b}^{\prime}\right)$ and $\left(\mathrm{c}^{\prime}\right)$ are equivalent each other: $\left(\mathrm{a}^{\prime}\right) J_{k}<\infty$; $\left(\mathrm{b}^{\prime}\right) \lim _{r \rightarrow \infty} h_{1 k}(r)<\infty$; and $\left(\mathrm{c}^{\prime}\right) \lim _{r \rightarrow \infty} h_{j k}(r)<\infty$ for any $j=1,2, \cdots$.

(iv) $\lim _{r \rightarrow \infty} f_{j k}(r) / f_{0 k}(r)=\left[\lim _{r \rightarrow \infty} h_{j k}(r)\right]^{-1}$ for any $j$ and $k$.

Proof. We obtain that

$$
\begin{aligned}
& h_{j k}(r)=\left(\lambda_{j}-\lambda_{0}\right) \int_{0}^{r} G(r, t) h_{j k}(t) d t, \\
& G(r, t)=\int_{t}^{r} t^{-2}(t / s)^{n-1}\left[g_{0 k}(t) / g_{0 k}(s)\right]^{2} d s .
\end{aligned}
$$

By using (6.27) we can show the lemma in the same way as in the proof of Lemma 6.8 .

Q.E.D.

Lemma 6.10. Suppose that $I_{2}<\infty$. Then $I_{1}<\infty\left(\right.$ or $\left.I_{1}=\infty\right)$ if and only if $J_{k}<\infty\left(\right.$ or $\left.J_{k}=\infty\right)$ for all $k=0,1, \cdots$.

Proof. Lemma 6.8 implies that if $I_{2}<\infty$, then for any $k$ there exists a positive constant $C$ such that

$$
C^{-1} \leqq g_{00}(r) / g_{0 k}(r) \leqq C \quad \text { for } \quad 1<r<\infty .
$$

Since $J_{0}=I_{1}$, this together with (6.26) shows that

$$
C^{-4} I_{1} \leqq J_{k} \leqq C^{4} I_{1}
$$

which proves the lemma.

Q.E.D.

Proof of Theorem 6.4. Let $H_{k}\left(x, x^{\prime}\right), k=1,2, \cdots$, be the minimal Green's function for $\left(L+\mu_{k} W, E\right)$ given by (6.7). Write $x=(r, \omega)$ and $x^{\prime}=\left(r^{\prime}, \omega^{\prime}\right)$. By (3.10), for any $\delta>0$ there exists a positive constant $C$ such that

$$
C \leqq G_{k}\left(x, x^{\prime}\right) \equiv \frac{H_{k}\left(x, x^{\prime}\right)}{g_{0 k}(r) f_{0 k}\left(r^{\prime}\right) \varphi_{0}(\omega) \varphi_{0}\left(\omega^{\prime}\right)} \leqq C^{-1}
$$


for any $r^{\prime} \geqq \max (1, r+\delta)$ and $\left(\omega, \omega^{\prime}\right)$ in $D^{2}$. Thus we obtain by Lemma 5.13 that

$$
0 \leqq \lim _{x^{\prime} \rightarrow \infty d} \frac{H_{k}\left(x, x^{\prime}\right)}{H_{0}\left(x_{0}, x^{\prime}\right)} \leqq C^{-2} \lim _{r^{\prime} \rightarrow \infty} \frac{g_{0 k}(r) f_{0 k}\left(r^{\prime}\right) \varphi_{0}(\omega)}{g_{00}(1) f_{00}\left(r^{\prime}\right) \varphi_{0}\left(\omega_{0}\right)}=0
$$

Thus with the same notation as in Theorem 3.5, $K_{k}(x, \infty d)=0$ for any $k=1,2, \cdots$. For $(s, \eta)$ in $(\max (r, 1), \infty) \times \partial D$,

$$
\lim _{x^{\prime} \rightarrow(s, \eta)} \frac{H_{k}\left(x, x^{\prime}\right)}{H_{0}\left(x_{0}, x^{\prime}\right)}=\frac{G_{k}(x,(s, \eta)) g_{0 k}(r) \varphi_{0}(\omega) f_{0 k}(s)}{G_{0}\left(x_{0},(s, \eta)\right) g_{00}(1) \varphi_{0}\left(\omega_{0}\right) f_{00}(s)} .
$$

Thus $K_{k}(x,(s, \eta))>0$ for any $k=1,2, \cdots$. By (6.28),

$$
K_{k}(x,(s, \eta)) \longrightarrow K_{k}(x, \infty d) \quad \text { as }(s, \eta) \rightarrow \infty d .
$$

In this way we can show (P. I), (P. II) and (P. III) with $\Sigma_{00}=\{\infty d\}$ and $\Sigma_{0 \infty}=\partial E$. Hence Theorem 3.5 implies Theorem 6.4.

Q.E.D.

Proof of Theorem 6.6. The same argument as in the proof of Theorem 6.4 shows that (P. I), (P. II) and (P. III) hold with $\Sigma_{00}=\infty \bar{D}$ and $\Sigma_{0 \infty}=\partial E$. Thus Theorem 6.6 follows from Theorem 3.5 and Lemma 6.9.

Q.E.D.

Proof of Theorem 6.5. Lemmas 6.8 6.10 and Theorem 5.3 imply that $a_{0 k}, k=0,1, \cdots$, defined by $(6.15)$ are positive and

$$
\begin{aligned}
& \lim _{x^{\prime} \rightarrow \infty d} H_{k}\left(x, x^{\prime}\right) / H_{0}\left(x_{0}, x^{\prime}\right) \\
& =\lim _{\substack{r^{\prime} \rightarrow \infty \\
\omega^{\prime} \in D}} \frac{\sum_{j=0}^{\infty} g_{j k}(r)\left[f_{j k} / f_{0 k}\right]\left(r^{\prime}\right) \varphi_{j}(\omega)\left[\varphi_{j} / \varphi_{0}\right]\left(\omega^{\prime}\right) f_{0 k}\left(r^{\prime}\right)}{\sum_{j=0}^{\infty} g_{j 0}(1)\left[f_{j 0} / f_{00}\right]\left(r^{\prime}\right) \varphi_{j}\left(\omega_{0}\right)\left[\varphi_{j} / \varphi_{0}\right]\left(\omega^{\prime}\right) f_{00}\left(r^{\prime}\right)} \\
& =a_{0 k} g_{0 k}(r) \varphi_{0}(\omega) /\left[g_{00}(1) \varphi_{0}\left(\omega_{0}\right)\right] .
\end{aligned}
$$

Thus (P. I $) \sim\left(\mathrm{P}\right.$. III) hold with $\Sigma_{0 \infty}=\partial E+\{\infty d\}$, and so Theorem 6.5 follows from Theorem 3.5 .

Q.E. D.

Proof of Theorem 6.7. The same argument as in the proof of Theorem 6.5 shows that $(\mathrm{P}$. I $) \sim\left(\mathrm{P}\right.$. III) hold with $\Sigma_{0 \infty}=\partial E+\infty \bar{D}$; furthermore,

$$
\lim _{x^{\prime} \rightarrow \infty \xi} \frac{H_{k}\left(x, x^{\prime}\right)}{H_{0}\left(x_{0}, x^{\prime}\right)}=\frac{\sum_{j=0}^{\infty} a_{j k} g_{j k}(r) \varphi_{j}(\omega)\left[\varphi_{j} / \varphi_{0}\right](\xi)}{\sum_{j=0}^{\infty} a_{j 0} g_{j 0}(1) \varphi_{j}\left(\omega_{0}\right)\left[\varphi_{j} / \varphi_{0}\right](\xi)}
$$

Thus Theorem 6.7 follows from Theorem 3.5.

Q.E.D.

We conclude this section with several remarks. 
Remark 6.11. The results of this section can be extended to an operator (6.1) with $V$ and $W$ being principally radially symmetric along the line given in $[23$, Section 5$]$.

Remark 6.12. Aikawa [3] identified the Martin compactification of $E \times F$ for $(-\Delta, E \times F)$, where $E=R^{n}$ or $R_{+}^{n}$, and $F$ is a bounded Lipshitz domain in $R^{m}$. In this special case we see that $I_{1}<\infty$ and $I_{2}=\infty$. Thus his result is a special case of Theorem 6.6. Take, for example, $\left(-\Delta, R^{n} \times F\right)$. Then we obtain from Theorem 6.6 that

$$
\begin{aligned}
& \Sigma=\sigma=R^{n} \times \partial F+\left\{(\infty \xi, f) ; \xi \in S^{n-1}\right\}, \\
& K(x, \infty \xi, y, f)=\phi_{0}(y) \exp \left(\lambda_{0}^{1 / 2} x \xi\right) /\left[\psi_{0}\left(y_{0}\right) \exp \left(\lambda_{0}^{1 / 2} \omega_{0} \xi\right)\right] .
\end{aligned}
$$

Remark 6.13. Theorems 6.4 6.7 correspond to the following four cases I $\sim$ IV, respectively; i.e., I: $I_{1}=I_{2}=\infty$; II : $I_{1}=\infty$ and $I_{2}<\infty$; III : $I_{1}<\infty$ and $I_{2}=\infty ;$ IV $: I_{1}<\infty$ and $I_{2}<\infty$. These four cases can be realized by (6.1) with $W(x)=(1+|x|)^{b}$ on $R^{n} \times F$. More precisely, when $b>-2$, three cases I, III and IV can occour; when $b=-2$, two cases I and IV can do; and when $b<-2$, three cases I, II and IV can do.

\section{$\S 7$. Direct Sum Decomposition}

In this section we give a method to construct the Martin boundary for a domain by decomposing it into a finite union of domains which are disjoint outside a compact set.

In order to states a main result of this section we need some preparation. Let $L$ be a second order elliptic differential operator (1.1) satisfying (i) (iii) on a non-compact connected orientable Riemannian manifold $M$ of dimension $n$ and class $C^{2}$. Let $\Omega$ and $\Omega_{j}, j=0,1, \cdots, \nu$ ( $\nu$ : a natural number), be Lipshitz domains of $M$ such that

$$
\Omega=\bigcup_{j=0}^{\nu} \Omega_{j}
$$

$\bar{\Omega}_{0} \quad$ is compact, $\quad \bar{\Omega}_{\jmath} \cap \bar{\Omega}_{j}=\phi \quad(i \neq j ; i, j=1, \cdots, \nu)$, and $\quad \Omega \neq \phi \quad(j=1, \cdots, \nu)$. Suppose that $(L, \Omega)$ is subcritical. Let $G, \Sigma, \sigma$ and $K$ be the minimal Green's function, Martin boundary, minimal Martin boundary, and Martin kernel for $(L, \Omega)$ with reference point $x_{0}$. By Theorems 1.5 and $1.7,\left(L, \Omega_{\jmath}\right)$ is also subcritical for $j=1, \cdots, \nu$. Denote by $G_{j}, \Sigma_{j}, \sigma_{j}$ and $K_{j}$ those corresponding to $\left(L, \Omega_{j}\right)$ with reference point $x_{j}$. In view of Theorem 2.1 , put

$$
\Gamma=\Sigma \backslash \partial \Omega, \quad \gamma=\sigma \backslash \partial \Omega, \quad \Gamma_{\jmath}=\Sigma_{j} \backslash \partial \Omega_{j} \quad \text { and } \quad \gamma_{j}=\sigma_{j} \backslash \partial \Omega_{j} .
$$

Let $H_{+}(L, \Omega, \Gamma)$ be a metric space defined by

$$
H_{+}(L, \Omega, \Gamma)=\left\{u \in H_{1 \mathrm{loc}}^{1}(\Omega) ; L u=0 \text { and } u \geqq 0 \text { in } \Omega,\left.u\right|_{\partial \Omega}=0\right\}
$$


with the metric associated with the uniform convergence on each compact subset of $\Omega$. Denote by $H_{+}\left(L, \Omega_{j}, \Gamma_{j}\right)$ the one corresponding to $\left(L, \Omega_{j}\right)$, $j=1, \cdots, \nu$. We shall write $H_{+}=H_{+}(L, \Omega, \Gamma)$ and $H_{j+}=H_{+}\left(L, \Omega_{j}, L_{\jmath}\right)$ when there is no fear of confusion. It is clear that for any $\xi$ in $\Gamma$ (or $\xi$, in $\Gamma_{j}$ ) $K(\cdot, \xi)$ (or $K_{\jmath}\left(\cdot, \xi_{j}\right)$ ) belongs to $H_{+}$(or $H_{j+}$, respectively).

Now, let us introduce a mapping $T$ from $H_{+}$to the direct sum

$$
\bigoplus_{j=1}^{\nu} H_{+}\left(L, \Omega_{\jmath}, \Gamma_{j}\right)
$$

of $H_{1+}, \cdots, H_{\nu+}$. Let $u$ be in $H_{+}$. For $j=1, \cdots, \nu$, choose a nonnegative function $\varphi_{3}$ in $C_{0}^{2}(M)$ such that $\varphi_{\jmath}=1$ in a neighborhood of $\overline{\partial \Omega, \cap \Omega}$. Set

$$
B, u(x)=(\varphi, u)(x)-\int_{\Omega_{j}} G_{\jmath}(x, y) L(\varphi, u)(y) d y
$$

for $x$ in $\Omega$. Clearly, $B_{\jmath} u$ is a unique positive solution of the Dirichlet problem: $L v=0$ in $\Omega_{\jmath}, v=u$ on $\partial \Omega_{\jmath}$, and $v$ is of minimal growth at $\Gamma_{\jmath}$. Let $u_{\jmath}=\left.u\right|_{\Omega_{\jmath}}$. Define a mapping $T$ from $H_{+}$to the direct sum by

$$
T u=\left(u_{1}-B_{1} u, u_{2}-B_{2} u, \cdots, u_{\nu}-B_{\nu} u\right) .
$$

Next, we introduce a mapping $S$ from the direct sum to $H_{+}$. (We shall show later that $S$ is the inverse of $T$.) Let $v=\left(v_{1}, \cdots, v_{\nu}\right)$ with $v_{\jmath} \in H_{\jmath+}$, and $v_{\jmath}^{\sim}$ the extension of $v_{\jmath}$ on $\Omega_{\jmath}$ to $M$ defined by $v_{\jmath}^{\sim}=0$ on $M \backslash \Omega_{j}$. Since $v_{j}$ vanishes Hölder continuously on $\partial \Omega$, and there exists a positive constant $C$ such that

$$
\int_{\Omega_{j}} \alpha\left|\nabla v_{j}\right|^{2} d x \leqq C \int_{\Omega_{j}}|\nabla \alpha|^{2} v_{j}^{2} d x
$$

for all $\alpha \geqq 0$ in $C_{0}^{1}\left(\Omega_{\jmath} \cap N\right)$ with $N$ being a sufficiently small neighborhood of a point of $\partial \Omega_{\jmath}$ (see [33, Lemma 5.2]), $v_{j}^{\sim}$ belongs to $H_{\text {loc }}^{1}(M)$. Thus the restriction $V$, of $L v_{\jmath}^{\sim}$ to $\Omega$ belongs to $H^{-1}(\Omega)$. Furthermore, we can show that $V_{\jmath} \leqq 0$ in the distribution sense as follows: (i) Choose a positive solution $h$ of $L h=0$ in a neighborhood of $\overline{\partial \Omega_{\jmath} \cap \Omega}$; (ii) use the identity $h V_{j}=L_{h}\left[\left(v_{j} / h\right)^{\sim}\right]$, where $L_{h}$ is the operator given by (1.7); and (iii) show that $L_{h}\left[\left(v_{j} / h\right)^{\sim}\right] \leqq 0$ by applying Lemma 2.10 of $[1]$. We are now ready to define $S$ by

$$
S v(x)=\sum_{j=1}^{\nu}\left(v_{\jmath}^{\sim}(x)-\int_{\Omega} G(x, y) L \tilde{v_{j}}(y) d y\right) .
$$

We snall denote by $G^{*} L v_{j}^{\tilde{j}}$ the second term in the right hand side of (7.5). The above argument shows that $-G^{*} L v_{j}^{\tilde{j}}$ multiplied by any nonnegative function in $C_{0}^{2}(M)$ is a nonnegative function in $H_{0}^{1}(\Omega)$. Thus $S$ is a mapping from the direct sum to $H_{+}$. 
Theorem 7.1. Suppose that $(L, \Omega)$ is subcritical and $\Omega$ is decomposed as (7.1). Then one has:

(i) $S$ is a continuous order preserving bijection from the direct sum $\bigoplus_{\jmath=1}^{\nu} H_{+}\left(L, \Omega_{\jmath}, \Gamma_{\jmath}\right)$ to $H_{+}(L, \Omega, \Gamma) ; T$ is a continuous order preserving bijection from $H_{+}$to the direct sum; $T=S^{-1}$ and $S=T^{-1}$.

(ii) There exists a homeomorphism $\Phi$ from $\Gamma$ to the topological union $\sum_{j=1}^{\nu} \Gamma_{\mathrm{J}}$ with $\Gamma_{\imath} \cap \Gamma_{\mathrm{J}}=\phi(i \neq j)$. Furthermore, for any $\xi$ in $\Gamma$ with $\Phi(\xi) \in \Gamma_{k}$, if $y_{\jmath} \rightarrow \xi$ as $j \rightarrow \infty$ in $\bar{\Omega}^{L}$, then $y_{\jmath} \rightarrow \Phi(\xi)$ as $j \rightarrow \infty$ in $\bar{\Omega}_{k}^{L}$.

(iii) $\Phi$ maps $\gamma$ onto $\sum_{j=1}^{\nu} \gamma_{\jmath}$.

(iv) For any $\xi$ in $\Gamma$ such that $\Phi(\xi)$ belongs to $\Gamma_{k}$,

$$
K(x, \xi)=S\left(\bigoplus_{\jmath=1}^{\nu} v_{j, \xi}\right)(x) / S\left(\bigoplus_{\jmath=1}^{\nu} v_{\jmath, \xi}\right)\left(x_{0}\right),
$$

where $v_{\jmath, \xi}=0$ for $j \neq k$ and $v_{k, \xi}(x)=K_{k}(x, \Phi(\xi))$.

Proof. (i ) Suppose that a sequence $\left\{v^{k}\right\}_{k=1}^{\infty}$ in the direct sum converges to $v$. Then $\left\{\left(v_{\jmath}^{k}\right)^{\sim}\right\}_{k}$ converges to $v_{\tilde{j}}$ in $H_{\mathrm{loc}}^{1}(M)$, and $\left\{L\left(v_{\jmath}^{k}\right)^{\sim}\right\}_{k}$ converges to $L v_{j}$ in $H^{-1}(\Omega)$. Thus $\left\{S v^{k}\right\}_{k}$ converges to $S v$ in $H_{+}$, which proves the continuity of $S$. If $w \geqq v$ in the direct sum, then $S(w-v) \geqq 0$; which implies that $S w \geqq S v$. Hence $S$ is order preserving. Similarly, we obtain that $T$ is continuous and order preserving. Let us show that $T S v=v$ for any $v$ in the direct sum. With $w=-\sum_{\jmath=1}^{\nu} G^{*} L v_{\tilde{j}}$, we have that

$$
(T S v)_{k}=\left.\sum_{j=1}^{\nu} v_{j}^{\sim}\right|_{\Omega_{k}}+\left.w\right|_{\Omega_{k}}-B_{k}\left(\sum_{\jmath=1}^{\nu} v_{j}^{\sim}+w\right)=v_{k}+\left.w\right|_{\Omega_{k}}-B_{k} w .
$$

We claim that $W \equiv w-B_{k} w=0$ in $\Omega_{k}$. Since $W=0$ on $\partial \Omega_{k}$ and $W$ is of minimal growth at $\Gamma_{k}$, we see that $W$ is a nonnegative solution of $L u=0$ in $\Omega_{k}$ which is of minimal growth at $\Sigma_{k}$. Thus Theorem 1.6 implies that $W=0$ in $\Omega_{k}$, which shows that $(T S v)_{k}=v_{k}$ for any $k$. In order to complete the proof of (i) it suffices to show that $T$ is one to one. Suppose that $T u=T v$ for $u$ and $v$ in $H_{+}$. Since $(u-v)_{\jmath}=B_{\jmath}(u-v)$ for any $j=1, \cdots, \nu$ and $u-v=0$ on $\partial \Omega$, we obtain that

$$
(u-v)(x) \leqq \varepsilon G(x, y)
$$

for any $\varepsilon>0$, where $y$ is a fixed point in $\Omega$. Thus $u \leqq v$ in $\Omega$. Similarly, $v \leqq u$. Hence $u=v$, which proves that $T$ is one to one.

(ii) We have that

$$
G_{k}(x, y)=G(x, y)-B_{k} G(x, y),
$$

which implies that

$$
G_{k}(x, y) / G\left(x_{0}, y\right)=K(x, y)-B_{k} K(x, y) .
$$

Suppose that $\left\{y_{j}\right\}_{j=1}^{\infty}$ is a sequence in $\Omega_{k}$ such that $\lim _{j \rightarrow \infty} y_{j}=\xi \in \Gamma$ in $\bar{\Omega}^{L}$. Then, by (7.8), there exists a unique $\xi_{k}$ in $\Gamma_{k}$ such that $y_{j} \rightarrow \xi_{k}$ in $\bar{\Omega}_{k}^{L}$ as $j \rightarrow \infty$, $K(x, \xi)-B_{k} K(x, \xi)>0$ in $\Omega_{k}$, and 


$$
K_{k}(x, \xi)=\left[K(x, \xi)-B_{k} K(x, \xi)\right] /\left[K\left(x_{k}, \xi\right)-B_{k} K\left(x_{k}, \xi\right)\right] .
$$

Since $K\left(x, y_{\jmath}\right), j \gg 1$, are positive solutions of $L u=0$ in $\Omega_{l}(l \neq k)$ which are of minimal growth at $\Gamma_{l}$ and uniformly bounded on each compact subset of $\bar{\Omega}_{l}$, we further obtain that

$$
K(x, \xi)-B_{l} K(x, \xi)=0 \text { in } \Omega_{l}, l \neq k .
$$

The equalities (7.9) and (7.10) show that if a sequence $\left\{y_{\jmath}\right\}_{j=1}^{\infty}$ in $\Omega$ converges to $\xi$ in $\Gamma$, then there exists a unique pair $\left(k, \xi_{k}\right)$ with $\xi_{k}$ in $\Gamma_{k}$ such that $y_{\jmath} \rightarrow$ $\xi_{k}$ as $j \rightarrow \infty$ in $\bar{\Omega}_{k}^{L}$. If we define $\Phi$ by $\Phi(\xi)=\xi_{k}$, we get (ii).

(iii) Since $S$ and $T$ are order preserving, (iii) follows from (7.9).

(iv) follows from (i) and (7.9).

Q.E.D.

A direct but interesting consequence of Theorem 7.1 is the following theorem which asserts, for example, that the Martin boundary over infinity is stable under compact perturbation in a sense.

Theorem 7.2. Suppose that $(L, \Omega)$ and $\left(L^{\prime}, \Omega^{\prime}\right)$ satisfy the hypotheses in Theorem 7.1; and assume that $\Omega_{\jmath}=\Omega_{j}^{\prime}$ for $j \neq 0$ and $L=L^{\prime}$ on $\cup_{\jmath=1}^{\nu} \Omega_{\jmath}$. Let $\Gamma$, $\gamma$ and $K$ be as in Theorem 7.1; and $\Gamma^{\prime}, \gamma^{\prime}$ and $K^{\prime}$ the ones corresponding to $\left(L^{\prime}, \Omega^{\prime}\right)$. Then $\Gamma=\Gamma^{\prime}, \gamma=\gamma^{\prime}$, and there exists a positive constant $C$ such that

$$
C \leqq K(x, \xi) / K^{\prime}(x, \xi) \leqq C^{-1}
$$

for all $\xi$ in $\Gamma$ and $x$ in $\cup_{j=1}^{\nu} \Omega_{j}$.

Proof. (7.11) follows from the Harnack inequality, the comparison theorem, and (7.6).

Q.E. D.

Remark. In the special case where $\nu=1$ and $\partial \Omega_{1} \subset \Omega \cap \Omega^{\prime}$, results relevant to Theorem 7.2 were given in [23, Section 2], [24], [21], [27] and [37] (see also [32]).

We conclude this section with a remark and examples illustrating the scope of Theorem 7.1. Systematic applications of Theorem 7.1 are left to the reader.

Example 7.3. Let $D$ be a Lipshitz domain whose closure is included in the upper hemi-sphere, and

$$
\Omega=\left\{x \in R^{n} \backslash\{0\} ; x /|x| \in D\right\} \cup R^{n-1} \times(-1,1) .
$$

Then we see that the spectrum of the Dirichlet realization $(-\Delta) \widetilde{\Omega}$ of $-\Delta$ in $L_{2}(\Omega)$ consists of $[0, \infty)$. We might expect that positive harmonic functions in $\Omega$ vanishing on $\partial \Omega$ are proportional as in the bounded domain case. However Theorems 7.1, 6.6 and 5.4 (see also Remarks 5.7 and 6.12) show that 
$\sigma \backslash \partial \Omega$ is homeomorphic to $\{\infty d\}+S^{n-1}$,

where $\sigma$ is the minimal Martin boundary for $(-\Delta, \Omega)$.

Example 7.4. Let $F$ be a bounded Lipshitz domain in $R^{m}$, and $\lambda_{0}$ the smallest eigenvalue of $(-A)_{\tilde{F}}$, where $A$ is the Laplace operator on $R^{m}$. Let $\Omega=R^{1} \times F$ and $\Omega^{\prime}=\Omega \backslash B$, where $B$ is a closed ball in $\Omega$. Then Theorem 3.3 shows that $\left(-\Delta-\lambda_{0}, \Omega\right)$ is critical and any positive harmonic function is a constant multiple of $\psi_{0}(y)$, where $\psi_{0}$ is an eigenfunction for $\lambda_{0}$. On the other hand, Theorem 1.8 shows that $\left(-\Delta-\lambda_{0}, \Omega^{\prime}\right)$ is subcritical; and we see from Theorems 7.1 and 3.5 that any positive harmonic function in $\Omega^{\prime}$ vanishing on $\partial \Omega^{\prime}$ is a nonnegative linear combination of two positive harmonic functions $K_{ \pm}$vanishing on $\partial \Omega^{\prime}$ such that

$$
\begin{aligned}
K_{ \pm}(x, y) & =[|x|+O(1)] \psi_{0}(y) \quad \text { as } \quad x \rightarrow \pm \infty \\
& =\left[C_{上}+O\left(|x|^{-1}\right)\right] \psi_{0}(y) \text { as } \quad-x \rightarrow \pm \infty,
\end{aligned}
$$

where $C_{\Perp}$ are positive constants (cf. Theorem A.7 and Example A.10 of [23]).

Remark 7.5. In view of Theorem 2.1 one might expect that there holds such a more localized version of Theorem 7.1 as the following:

Suppose that $(L, \Omega)$ is subcritical. Let $\Omega^{\prime}$ be a Lipshitz subdomain of $\Omega$. Denote by $\Sigma$ and $\Sigma^{\prime}$ the Martin boundary for $(L, \Omega)$ and $\left(L, \Omega^{\prime}\right)$, respectively. Then $\Sigma^{\prime} \backslash \partial \Omega^{\prime}$ is imbedded into $\Sigma \backslash \partial \Omega$.

But this is not valid in general as the following example shows. Let $\Omega=$ $R^{2}, \Omega^{\prime}=\left\{x \in R^{2} ; x_{1}>0\right.$ and $\left.x_{2}>0\right\}$, and $L=-\Delta+V$, where $V=1$ on $\Omega^{\prime}$ and $V=0$ on $\Omega \backslash \bar{\Omega}^{\prime}$. Then we see that $\Sigma^{\prime} \backslash \partial Q^{\prime}$ is homeomorphic to $[0, \pi / 2]$. On the other hand, Theorem 7.1 of [23] implies that $\Sigma$ consists of one point.

\section{Appendix. A Uniqueness Theorem}

In this appendix we give a uniqueness theorem for positive solutions of an elliptic equation in a cone or $R^{n}$.

Let $L$ be an elliptic operator (1.1) on $M=R^{n}, n \geqq 2$, satisfying (i ) (iii), and the following condition:

There exist positive constants $\alpha<1<\beta, \gamma, \lambda, \Lambda$, and a sequence $\left\{R_{k}\right\}_{k=1}^{\infty}$ of positive numbers with $R_{k} \rightarrow \infty$ as $k \rightarrow \infty$ such that for all $k=1,2, \cdots$

$$
\begin{gathered}
\lambda|\xi|^{2} \leqq \sum_{i, j=1}^{n} a_{i j}(x) \xi_{\imath} \xi_{j} \leqq \Lambda|\xi|^{2}, \quad x \in A_{k}, \quad \xi \in R^{n}, \\
\sum_{i=1}^{n}\left\||x|^{1-n / p}\left\{\left|b^{i}(x)\right|+\left|d^{i}(x)\right|\right\}\right\|_{L_{p}\left(A_{k}\right)} \\
+\left\||x|^{2-2 n / p} c(x)\right\|_{L_{p / 2}\left(A_{k}\right)} \leqq \gamma,
\end{gathered}
$$

where $A_{k}=\left\{x \in R^{n}: \alpha R_{k}<|x|<\beta R_{k}\right\}$. 
Let $D$ be a Lipshitz domain with nonempty boundary in the unit sphere $S^{n-1}$ of $R^{n}$. Let $E=R^{n}$ or a Lipshitz domain in $R^{n}$ of the form

$$
E=\left\{x \in R^{n} \backslash\{0\} ; x /|x| \in D\right\} \cup F,
$$

where $F$ is a bounded Lipshitz domain.

We consider the following problem:

$$
L u=0 \text { and } u>0 \text { in } E, u=0 \text { on } \partial E \text { (if } \partial E \neq \phi \text { ). }
$$

Recall that a positive solution of $L u=0$ in $E$ exists if and only if $\Gamma(L, E) \geqq 0$ (see Theorem 1.5 in Section 1).

Theorem A. Suppose that $\Gamma(L, E) \geqq 0$. Then (A.3) has a solution which is unique up to a constant multiple.

Proof. By virtue of Theorem 7.2, it suffices to show the theorem in the case where $E=R^{n}$ or a cone generated by $D$ (i. e., $F=\phi$ ). We treat only the cone case, since the other case can be treated similarly and more easily. Let $x_{0}$ be a fixed point in $E$ with $\left|x_{0}\right|=1$. For $r>1$, put $E_{r}=E \cap\{|x|<r\}$. Since $\Gamma\left(L, E_{r}\right)>0$ by Theorem 1.5 , there exists a positive solution $w_{r}$ satisfying

$$
L w_{r}=0 \text { in } E_{r}, w_{r}=0 \text { on } \partial E \cap E_{r}, w_{r}=1 \text { on } E \cap\{|x|=r\} .
$$

Put $u_{r}(x)=w_{r}(x) / w_{r}\left(x_{0}\right)$. By the Harnack inequality, there exists $\left\{r_{j}\right\}_{j=1}^{\infty}$ such that $r_{j} \rightarrow \infty$ as $j \rightarrow \infty$ and $\left\{u_{r_{j}}\right\}_{j}$ converges uniformly to a continuous function $u$ on each compact subset of $E$. Clearly, $u$ is a solution of (A.3). This proves the existence.

Let us show the uniqueness by applying Lemma 2.2 in Section 2. Let $\mathscr{F}$ denote the set of all solutions $u$ of (A.3) normalized by $u\left(x_{0}\right)=1$. Then the condition (ii) in Lemma 2.2 holds clearly. Suppose that $u$ and $v$ are solutions of (A.3). For $k=1,2, \cdots$, write $u_{k}(x)=u\left(R_{k} x\right)$. Then $u_{k}$ and $v_{k}$ are positive solutions of

$$
L_{k} w=0 \text { in } G \equiv\{x \in E ; \alpha<|x|<\beta\}, \quad w=0 \text { on } \partial G \cap \partial E,
$$

where

$$
L_{k}=-\sum_{i, j=1}^{n} \partial_{j}\left(a^{i j}\right)_{k} \partial_{i}+\sum_{i=1}^{n} R_{k}\left[\left(b^{i}\right)_{k} \partial_{i}-\partial_{i}\left(d^{i}\right)_{k}\right]+R_{k}^{2} c_{k} .
$$

We see that for all $k$

$$
\begin{gathered}
\lambda|\xi|^{2} \leqq \sum_{i, j=1}^{n}\left(a_{i j}(x)\right)_{k} \xi_{i} \xi_{j} \leqq \Lambda|\xi|^{2}, \quad x \in G, \quad \xi \leqq R^{n}, \\
\sum_{i=1}^{n}\left\{\left\|R_{k}\left(b^{i}\right)_{k}\right\|_{p}+\left\|R_{k}\left(d^{i}\right)_{k}\right\|_{p}\right\}+\left\|R_{k}^{2} c_{k}\right\|_{p / 2} \leqq C \gamma,
\end{gathered}
$$

where $\|\cdot\|_{p}$ denotes the norm of $L_{p}(G)$ and $C$ is a constant independent of $k$. 
Thus the comparison theorem (Theorem 1.3) and the Harnack inequality yield the inequality

$$
u\left(R_{k} x\right) / u\left(R_{k} x_{0}\right) \leqq C v\left(R_{k} x\right) / v\left(R_{k} x_{0}\right)
$$

for all $k$ and $x \subseteq\{x \in E ; 8|| x|-1|<\min (1-\alpha, \beta-1)\}$. Thus (2.1) holds with $O_{k}=E_{R_{k}}$ and $x_{k}=R_{k} x_{0}$. On the other hand, (2.2) holds because $u=v=0$ on $\partial E \cap E_{R_{k}}$ and $\Gamma\left(L, E_{R_{k}}\right)>0$. Hence Lemma 2.2 yields the theorem. Q.E.D.

Remark. In the case where $E$ is a cone with nonempty boundary, Theorem $A$ is essentially a considerable extension of Theorem 2.1 of [19]. The theorem in the special case $E=R^{n}$ includes Theorems 5.6 and 5.7 of [23] except for the asymptotic behavior at infinity of a positive solution. Together with results of [24], it also yields an extension of Theorem of [13].

Remark. Theorems A and 2.1 imply that the Martin compactification of $E$ for $(L, E)$ is the closur of $E$ in the one point compactification of $R^{n}$.

\section{References}

[1] Agmon, S., On positivity and decay of solutions of second order elliptic equations on Riemannian manifolds, in: D. Greco ed., Methods of Functional Analysis and Theory of Elliptic Equations, Liguori Editore, Naples (1982), 19-52.

[2] - On positive solutions of elliptic equations with periodic coefficients in $R^{n}$, spectral results and extensions to elliptic operators on Riemannian manifolds, in: I. W. Knowles and R. T. Lewis eds., Proc. Int. Conf. on Differential Equations, North-Holland Mathematical Studies, 22 (1984), 7-17.

[3] Aikawa, H., On the Martin boundary of Lipshitz strips, J. Math. Soc. Japan, 38 (1986), 527-541.

[4] Allegretto, W., Criticality and the $\lambda$-property for elliptic equations, J. Differential Eq., 69 (1987), 39-45.

[5] Ancona, A., Negatively curved manifolds, elliptic operators, and the Martin boundary, Ann. Math., 121 (1985), 429-461.

[6] Anderson, M.T. and Shoen, R., Positive harmonic functions on compact manifolds of negative curvature, Ann. Math., 121 (1985), 429-461.

[7] Caffarelli, L.A., Fabes, E., Mortola, S. and Salsa, S., Boundary behavior of nonnegative solutions of elliptic operators in divergence form, Indiana Univ. Math. J., 30 (1981), 621-640.

[8] Caffarelli, L.A. and Littman, W., Representation formulas for solutions to $\Delta u-u$ $=0$ in $R^{n}$, in: Studies in Partial Differential Equations, MAA Studies in Math., 23, Math. Association of America, Washington (1982), 249-263.

[9] Dahlberg, B.E.J., Estimates of harmonic measure, Arch. Rational Mech. Anal., 65 (1977), 272-288.

[10] Doob, J.L., Classical Potential Theory and Its Probabilistic Counterpart, SpringerVerlag, New York-Berlin-Heiderberg-Tokyo, 1984.

[11] Herglotz, G., Uber Potenzreihen mit positivem reelem Teil im Einheitskreis, Ber. Verhandl. Sächs. Akad. Wiss. Leipzig Math. Phys. Klasse, 63 (1911), 501-511.

[12] Hunt, R.A. and Wheeden, R.L., Positive harmonic functions on Lipshitz domains, 
Trans. Amer. Math. Soc., 147 (1970), 507-527.

[13] Imai, H., Picard principle for linear elliptic differential operators, Hiroshima Math. J., 14 (1985), 527-535.

[14] Imai, H. and Tada, T., Picard principle for rotation free densities on the Euclidean $N$-space $(N \geqq 3)$, Bull. Daido Inst. Tech., 13 (1978), 1-12.

[15] Itô, S., On existence of Green's function and positive superharmonic functions for elliptic operators of second order, J. Math. Soc. Japan, 16 (1964), 299-306.

[16] - Martin boundary for linear elliptic differential operators of second order in a manifold, J. Math. Soc. Japan, 16 (1964), 307-334.

[17] Jerison, D.S. and Kenig, C.E., Boundary behavior of harmonic functions in nontangentially accessible domains, Advances in Math., 46 (1982), 80-147.

[18] Karpelevic, F.I., The geometry of geodesics and the eigenfunctions of the BeltramiLaplace operator on symmetric space, Trudy Moscow Math. Obsc., 14 (1965), 48145; Trans. Moscow Math. Soc. (1965), 51-199.

[19] Landis, E.M. and Nadirashvili, N.S., Positive solutions of second order elliptic equations in unbounded domains, Math. USSR Sbornik, 54 (1986), 129-134.

[20] Li, P. and Tam, L.F., Positive harmonic functions on complete manifolds with non-negative curvature outside a compact set, Ann. Math., 125 (1987), 171-207.

[21] Loeb, P.A., An axiomatic treatment of pairs of elliptic differential equations, Ann. Inst. Fourier, 16 (1966), 167-208.

[22] Martin, R.S., Minimal positive harmonic functions, Trans. Amer. Math. Soc., 49 (1941), 137-172.

[23] Murata, M., Structure of positive solutions to $(-\Delta+V) u=0$ in $R^{n}$, Duke Math. J., 53 (1986), 869-943.

[24] - Isolated singularities and positive solutions of elliptic equations in $R^{n}$, Proc. Japan Acad., 63 Ser. A (1987), 146-148.

[25] - Structure of positive solutions to $(-\Delta+V) u=0$ in $R^{n}$, in: I. W. Knowles and Y. Saitō eds., Differential Equations and Mathematical Physics, Lecture Notes in Math., 1285, Springer-Verlag (1988), 342-347.

[26] Nakai, M., Martin boundary over an isolated singularity of rotation free density, J. Math. Soc. Japan, 26 (1974), 483-507.

[27] - Picard principle and Riemann theorem, Tôhoku Math. J. Second Ser., 28 (1976), 277-292.

[28] - and Tada, T., Extreme nonmonotoneity of Picard principle, Math. Ann., 281 (1988), 279-293.

[29] Phelps, R., Lectures on Choquet's theorem, Math. Studies 7, Van Nostrand, Princeton, N. J., 1965.

[30] Pinchover, Y., On positive solutions of elliptic and parabolic equations in $R^{n}$, C.R. Acad. Sc. Paris, Ser. I, 302 (1986), 447-450.

[31] - On positive solutions of elliptic equations with periodic coefficients in unbounded domains, in: P.W. Schaefer ed., Maximum Principles and Eigenvalue problems in Partial Differential Equations, Pitman Research Notes in Math., 175, Longman Press, (1988), 218-230.

[32] — - On positive solutions of second order elliptic equations, stability results and classification, Duke Math. J., 57 (1988), 955-980.

[33] Stampacchia, G., Le problème de Dirichlet pour les équations elliptique de second ordre à coefficients discontinuous, Ann. Inst. Fourier, 15 (1965), 189-257.

[34] Sur, M.G., The Martin boundary for a linear elliptic second-order operator, Izv. Acad. Nauk SSSR Ser. Math., 27 (1963), 45-60.

[35] Suzuki, N., Martin boundary for $\Delta-P$, Hiroshima Math. J., 14 (1984), 67-74.

[36] Tada, T., The Martin boundary for the half disk with rotation free densities, 
Hiroshima Math. J., 16 (1986), 315-325.

[37] Taylor, J.C., The Martin boundaries of equivalent sheaves, Ann. Inst. Fourier., 20 (1970), 433-456.

[38] - - On the Martin compactification of a bounded Lipshitz domain in a Riemann manifold, Ann. Inst. Fourier, 28 (1978), 25-52.

[39] Wu, J.M.G., Comparison of kernel functions, boundary Harnack principle and relative Fatou theorem on Lipshitz domains, Ann. Inst. Fourier (Grenoble), 28 (1978), 147-167. 
